The Astrophysical Journal Supplement Series, 35:239-261, 1977 October

(C) 1977. The American Astronomical Society. All rights reserved. Printed in U.S.A.

\title{
THE DYNAMICS AND HELIUM DISTRIBUTION IN HYDROGEN-HELIUM FLUID PLANETS
}

\author{
D. J. Stevenson* and E. E. Salpeter \\ Center for Radiophysics and Space Research and Physics Department, Cornell University \\ Received 1976 June 23; accepted 1977 April 13
}

\begin{abstract}
In the preceding paper (Paper I) we discussed the thermodynamic and microscopic transport properties of hydrogen-helium fluid mixtures. These results are used in the present paper for a semiquantitative analysis of the thermal and compositional history of an evolving hydrogenhelium planet such as Jupiter or Saturn.

First, the evolution of a homogeneous planet with no first-order phase transitions or immiscibilities is considered. The temperature gradient is at least adiabatic (sirce thermal conduction cannot transport a sufficient heat flux) and is also large enough to ensure that the fluid state prevails everywhere. Convection is therefore uninhibited by molecular viscosity, and the fractional superadiabaticity is very small, despite the inhibitory effects of rotation and magnetic field. Adiabatic, evolutionary models are discussed. The times taken for Jupiter and Saturn to reach their observed luminosities are about $4 \times 10^{9}$ and $2 \times 10^{9}$ years, respectively, essentially independent of formation details. The result for Saturn appears to be inconsistent with its actual age, assumed to be $\sim 4.5 \times 10^{9}$ years.

Next, the effects of a first-order molecular-metallic hydrogen transition are discussed for a pure hydrogen planet: A well-defined interface between the phases persists, despite the presence of convection. The temperature is continuous at the interface and the entropy is discontinuous, the change in entropy being equal to the latent heat of transition. Consequently, the heat content and derived "age" differ from that determined for a purely adiabatic model (by a factor between 1 and 2 , depending on the unknown latent heat).

Convection in the presence of a composition gradient is discussed, and the importance of overstable modes and diffusive-convective equilibria established. The convective transport of helium away from a localized helium source is shown to be inefficient because helium diffusivity is much less than heat diffusivity.

Evolutions with helium immiscibility (but no first-order molecular-metallic hydrogen transition) are discussed. Helium droplets nucleate from the supersaturated mixture, grow to $\sim 1 \mathrm{~cm}$ radius, and fall under the influence of gravity, despite the convection. Most of the energy release from this differentiation is available for radiation, and the decay time for the planet's excess luminosity is increased, typically by about a factor of 5 .

Finally, more complicated cases are discussed which include both immiscibility and the firstorder character of the molecular-metallic hydrogen transition. The Gibbs phase rule leads to a discontinuity of the helium fraction at the transition, the formation of a helium-rich core, and an energy release comparable to that for immiscibility. This core can grow at the expense of the helium content in either the metallic or molecular region. In some cases, the molecular envelope helium content is actually enhanced by upward convective transport of helium.

The various parameters (especially the critical temperature of the molecular-metallic hydrogen transition) are too uncertain for detailed quantitative conclusions. The success of adiabatic, homogeneous evolutionary calculations for Jupiter suggests that helium differentiation has not yet begun for that planet or has begun very recently $\left(\leqslant 10^{9}\right.$ years ago), which in turn suggests that the critical temperature for the molecular-metallic hydrogen transition cannot greatly exceed $20,000 \mathrm{~K}$. Helium differentiation in Saturn (and deviations from primordial abundance for helium and minor constituents in the atmosphere) appears to be required to explain the observed excess luminosity.
\end{abstract}

Subject headings: planets: abundances — planets: interiors - planets: Jupiter

\section{INTRODUCTION}

Modeling of the giant planets is a well-constrained problem and has reached a quite high level of sophistication in recent years. Present models of Jupiter
(Podolak and Cameron 1975; Zharkov and Trubitsyn 1976; Hubbard and Slattery 1976; Stevenson and Salpeter 1976; Podolak 1977) and Saturn (Podolak and Cameron 1974; Zharkov and Trubitsyn 1976) are substantially in agreement regarding the major 
features of these planets. However, none of these models systematically investigates the implications of the hydrogen-helium phase diagram. The hydrogen and helium are assumed to be uniformly mixed, and firstorder phase transitions are either assumed to not exist, or are inadequately treated. In the preceding paper (Stevenson and Salpeter 1977, hereafter Paper I) the phase diagram was discussed in detail, and in this paper, those results are applied to the thermal and compositional history of the hydrogen-helium planets.

Before outlining our approach to this problem, we summarize the main features of Jupiter and Saturn which are common to all the models referenced above. For Jupiter, these features are $(a)$ a composition that is roughly $65 \% \mathrm{H}, 30 \% \mathrm{He}$, and $5 \%$ other elements by mass, the latter being somewhat concentrated toward the center of the planet; $(b)$ an adiabatic temperature structure such that the temperature rises from about $180 \mathrm{~K}$ at $P=1$ bar, to about $10,000 \mathrm{~K}$ at $P \approx 3 \mathrm{Mbar}$ (the molecular-metallic hydrogen transition) and $20,000 \mathrm{~K}$ at the innermost hydrogen-helium region $(P \approx 45 \mathrm{Mbar})$; $(c)$ a metallic hydrogen-helium core that is 3 or 4 times more massive than the molecular envelope.

The main features for Saturn are less well established: (a) a composition of $50-55 \% \mathrm{H}, 20-25 \% \mathrm{He}$, and $15-20 \%$ other elements by mass, but with wider variations conceivable; $(b)$ an adiabatic temperature structure such that the temperature rises from about $140-150 \mathrm{~K}$ at $P=1$ bar to about $8500 \mathrm{~K}$ at $P \approx$ $3 \mathrm{Mbar}$ (the molecular-metallic hydrogen transition) and a central temperature of perhaps $\sim 11,000 \mathrm{~K} ;(c)$ a metallic hydrogen-helium core that is as little as one-third or as much as equal in mass to the molecular hydrogen envelope. For more details and comparisons for Jupiter and Saturn, see Stevenson (1977).

The main question we address in this paper is, Are the above models consistent with the hydrogen-helium phase diagram? In attempting to answer this, the following subsidiary questions necessarily arise:

1. Under what circumstances does a hydrogenhelium planet have an adiabatic thermal structure? Since the discovery of the excess infrared emission of Jupiter (Aumann et al. 1969; Ingersoll et al. 1976) and Saturn (Aumann et al. 1969; Nolt et al. 1974; Rieke 1975), it has been assumed that these planets are convective almost everywhere and hence adiabatic. However, this is not correct if there are first-order phase transitions or composition gradients.

2. Under what circumstances is a hydrogen-helium planet homogeneous? It is inevitable that some part of the planet will eventually evolve into a phase excluded region of the hydrogen-helium phase diagram, either because of the immiscibility or because of the Gibbs phase rule requirement that the helium content be discontinuous at the molecular-metallic hydrogen phase transition. The only doubt is whether this has occurred already, is occurring now, or will only occur in the future evolution of Jupiter or Saturn. Inhomogeneity is ensured for a temperature less than about $10,000 \mathrm{~K}$ at the molecular-metallic transition. The similarity between this and the actual temperature predicted by homogeneous models may not be a coincidence.

3. What implications does inhomogeneity have for the thermal evolution? Recent evolutionary calculations for Jupiter (Graboske et al. 1975; Hubbard 1977) appear capable of explaining the excess infrared emission as the release of primordial heat content from a homogeneous planet. A similar calculation for Saturn (Pollack et al. 1977) appears to be incapable of predicting sufficient heat flux after $4.5 \times 10^{9}$ years. However, if gravitational layering is possible, with the more dense helium separating toward the center of the planet, then a large energy source becomes available to augment the primordial heat content (Kiefer 1967; Salpeter 1973). Helium differentiation always occurs eventually, but the details are found to be quite complicated, in general. Approximate calculations indicate that the present luminosity of Saturn is readily explained by helium differentiation during the last $2 \times 10^{9}$ years.

4. What implications do the phase transitions have for the distribution of minor constituents (e.g., $\mathrm{H}_{2} \mathrm{O}$, $\mathrm{CH}_{4}, \mathrm{NH}_{3}$ ) ? Although we will not attempt a quantitative answer to this question, it is found from quite general considerations that the atmospheric composition is not in general representative of the bulk composition of the planet, even at levels deeper than any possible clouds. In view of the difficulty of estimating atmospheric helium abundance from remote observations, this fact may be the best observational test of our theory.

5. Can atmospheric observations be used to determine constraints on the thermal evolution of a fluid planet? The present distribution of constituents depends in a complicated way on the previous evolution of the planet. Unfortunately, we find that the current uncertainties in the hydrogen-helium phase diagram and transport properties preclude any firm predictions that relate the present compositional distributions to the past thermal evolution.

In this paper we proceed from the simple to the complex. In $\S$ II we discuss the particularly simple case of a homogeneous planet in which there are no first-order phase transitions. The assumption of homogeneity is common to almost all recent models of the evolution and internal structure of Jupiter. In this particular case, convective heat transport dominates almost everywhere, and the specific entropies of the atmosphere and deep interior are almost equal. Homogeneous, adiabatic evolutionary calculations then indicate that the times taken for Jupiter and Saturn to reach their observed excess luminosities are about $4 \times 10^{9}$ years and $2 \times 10^{9}$ years, respectively, essentially independent of the details of planetary formation.

In § III we discuss a pure hydrogen planet in which there is a fluid molecular hydrogen to fluid metallic hydrogen first-order phase transition. It is assumed that convection dominates the heat transport everywhere, except possibly near the pressures and temperatures corresponding to the phase transition. This general situation was considered in detail by Salpeter 


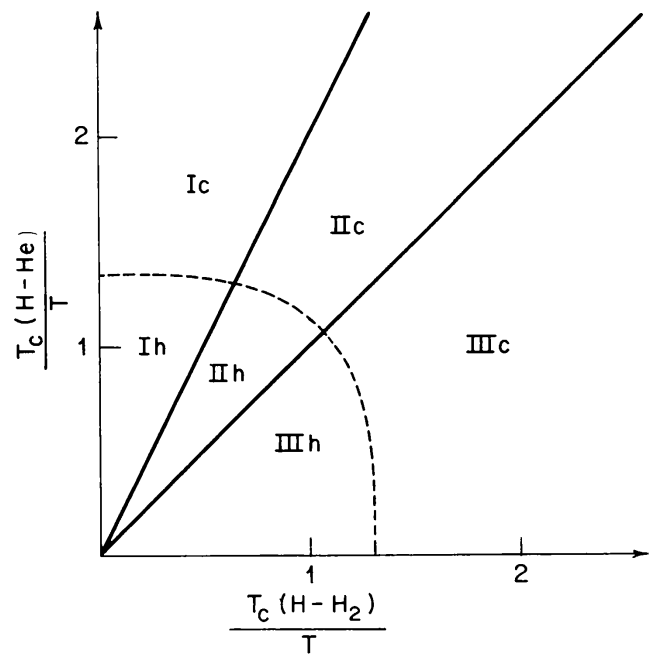

Fig. 1.-Various possible evolutionary regimes depending on the relative values of $T_{c}(\mathrm{H}-\mathrm{He}), T_{c}\left(\mathrm{H}_{2}-\mathrm{H}\right)$, and $T$. This figure assumes $T_{c}\left(\mathrm{H}_{2}-\mathrm{He}\right)=1 / 2 T_{c}(\mathrm{H}-\mathrm{He})$ and is the analog of Fig. 6 in Paper I. In Sector I, immiscibility effects dominate. In Sector III, the effects of the molecular-metallic hydrogen transition dominate. Sector II is intermediate and complicated (see text for discussion). The dashed line separates "hot" evolutions from "cold" evolutions.

and Stevenson (1976). We apply those considerations to Jupiter and Saturn, and conclude that a well-defined interface exists between the phases, strongly inhibiting convective flow in its vicinity. Since the temperature is essentially continuous across the interface, the entropies of the two phases are found to differ by the latent heat of the transition. Under these circumstances, the temperature in the metallic core can differ by up to a factor of 2 from that predicted for a fully adiabatic planet (but the actual factor is probably nearer unity than 2). A similar effect on the derived "age" of the planet is also predicted.

In $\S$ IV we discuss some general aspects of convection in the presence of compositional gradients. Particular attention is given to the most relevant case, in which thermal diffusion is greater than particle diffusion. Overstability and the convective transport of solute are discussed.

Sections V and VI are devoted to particular evolutionary sequences. In Figure 1, the various possibilities are characterized by the critical temperatures $T_{c}\left(\mathrm{H}-\mathrm{H}_{2}\right)$ and $T_{c}(\mathrm{H}-\mathrm{He})$, for the molecular-metallic hydrogen transition and the metallic hydrogen-helium mixture, respectively. This figure is directly analogous to Figure 6 of Paper I. As in that paper, we set $T_{c}\left(\mathrm{H}_{2}-\mathrm{He}\right)=$ $1 / 2 T_{c}(\mathrm{H}-\mathrm{He})$, where $T_{c}\left(\mathrm{H}_{2}-\mathrm{He}\right)$ is the critical temperature for the molecular mixture. The evolution of a planet can be characterized in Figure 1 by a straight line segment, the extension of which passes through the origin. Thus the evolution lies in one of the three sectors shown. For the purposes of our considerations, the starting point of the evolution is defined as the temperature of the central hydrogen-helium region of the planet, when that region first becomes degenerate (i.e., reaches megabar pressures). The dashed line in Figure 1 further subdivides the sectors according as to whether that starting point is "hot" or "cold." A "cold" situation is one in which a phase excluded region is encountered at the beginning of the evolution. A "hot" situation is one in which the evolutionary starting point is inside the dashed boundary. It is necessary to consider several possibilities, primarily because $T_{c}\left(\mathrm{H}-\mathrm{H}_{2}\right)$ is so uncertain (see the discussion in Paper I). There is also considerable uncertainty as to the starting temperature for the evolution.

In $\S \mathrm{V}$, Sector I of Figure 1 is considered. Since the immiscibility of helium in hydrogen is the main consideration here, this section assumes, for simplicity, that there is no first-order molecular-to-metallic hydrogen transition. It is also assumed that the starting point is "hot," since the starting temperature is likely to be well in excess of $T_{c}(\mathrm{H}-\mathrm{He}) \approx 1 \times 10^{4} \mathrm{~K}$. As the planet cools down, it becomes possible for droplets of helium-rich fluid to nucleate from the mixture, grow rapidly, and drift downward. The subsequent inhomogeneous evolution is discussed, using parameters appropriate to Jupiter and Saturn. Once this differentiation is initiated, a large energy source becomes available. Most of this energy is available for radiation. The rate at which the excess luminosity decreases with time is found to decrease by typically a factor of 5 relative to homogeneous evolution, once differentiation begins.

In $\S$ VI we discuss Sector III of Figure 1. The main consideration here is the first-order character of the molecular-metallic hydrogen transition, but helium insolubility is also an important consideration. Both "hot" and "cold" starting points are considered. In the "cold" case, the evolution depends on the relative densities of the coexisting helium-rich molecular phase and helium-poor metallic phase. If the former is more dense then there is a net downward transport of helium; if the latter is more dense then there is initially a small net upward transport of helium. We also discuss the "hot" case, in which there is always a net downward transport of helium.

Sector II in Figure 1 is not discussed in detail since there are no new effects in this sector that are not already present in Sector I or Sector III. The results for Sector II are, however, summarized in the concluding $\S$ VII. There, we summarize the various possible cases and their implications. A brief discussion of the disposition of minor constituents (such as water) is given, and some possible inadequacies in our analysis are assessed. Unfortunately, the uncertainties in the phase diagram and transport properties are still so great that we are unable to predict, say, the helium abundance in the Jovian and Saturnian atmospheres. However, the success of adiabatic, homogeneous evolutionary calculations for Jupiter suggest that helium differentiation has not yet begun for that planet, or has begun very recently $\left(\leqslant 10^{9}\right.$ years ago). Helium differentiation in Saturn appears to be required to explain its observed excess luminosity, but the uncertainties are large. 


\section{THE THERMAL EVOLUTION OF A HOMOGENEOUS PLANET}

We consider first the unlikely case where the molecular metallic hydrogen transition is not first-order and there is unlimited solubility of helium in hydrogen. The infrared excesses of Jupiter and Saturn led Hubbard $(1968,1973)$ to propose that such planets are convective almost everywhere, with the consequence that the specific entropies of the deep atmosphere and metallic interior are equal (i.e., the temperature and pressure are adiabatically related). This "adiabatic hypothesis" is based on three assertions: (i) The internal heat flux is too high to be transported by conduction (electronic, molecular, or radiative) at a subadiabatic temperature gradient. (ii) The resulting internal temperature is therefore high enough to ensure that the fluid state prevails everywhere. (iii) Convection is therefore not inhibited by viscosity and readily transports the required heat flux with only a very small superadiabaticity.

The inadequacy of electronic conduction has been discussed elsewhere (Stevenson and Ashcroft 1974; Stevenson and Salpeter 1976; Stevenson 1976) for the particular case of Jupiter. Similar calculations can be made for Saturn. In both cases, the thermal conductivity in the metallic core is about $2 \times 10^{8}$ ergs $\mathrm{cm}^{-1} \mathrm{~s}^{-1} \mathrm{~K}^{-1}$ (eq. [11], Paper I) and the adiabatic temperature gradient is typically $2 \times 10^{-6} \mathrm{~K} \mathrm{~cm}^{-1}$, so the conductive heat flux is typically $400 \mathrm{ergs} \mathrm{cm}^{-2}$ $\mathrm{s}^{-1}$. The total internal heat flux that emerges into the atmosphere is about $(7 \pm 2) \times 10^{3} \mathrm{ergs} \mathrm{cm}^{-2} \mathrm{~s}^{-1}$ for Jupiter (Ingersoll et al. 1976) and $(4 \pm 1.5) \times 10^{3} \mathrm{ergs}$ $\mathrm{cm}^{-2} \mathrm{~s}^{-1}$ for Saturn (Aumann et al. 1969; Nolt et al. 1974; Rieke 1975). In each case, the energy source must be gravitational (Hubbard and Smoluchowski 1973), but the distribution of the energy source is not accurately known. However, even for a highly decentralized energy source such as primordial heat, the heat flux at the molecular-metallic hydrogen transition is comparable to (and may even be larger than) the heat flux emerging into the atmosphere, because of the smaller surface area. In both planets, the inequality between conductive and total heat flux in the metallic region is not enormous, but is nevertheless strong enough to be almost certain. A smaller, purely conductive region near the center of each planet is not excluded.

In the molecular region, electronic or molecular conduction is negligible but radiative opacity could conceivably be low enough to allow a radiative rather than adiabatic thermal structure. However, the discussion in Paper I indicates that the opacity of pure hydrogen alone is sufficient to ensure convection, except at temperatures where the $1500 \mathrm{~cm}^{-1}$ to $3000 \mathrm{~cm}^{-1}$ window is important (i.e., $400 \mathrm{~K} \leqslant T \leqslant$ $700 \mathrm{~K})$. In this region, a solar abundance of "ices" $\left(\mathrm{H}_{2} \mathrm{O}, \mathrm{CH}_{4}, \mathrm{NH}_{3}\right)$ will probably "block" the window in the pure hydrogen spectrum. It follows that a deep radiative layer, almost immediately below the observable atmosphere, cannot be discounted until we know the abundance of minor constituents in such planets. It should be noted, however, that a radiative layer is not compatible with the interpretation by Gulkis and Poynter (1972) of the thermal radio emissions from Jupiter and Saturn. It would also be very difficult to reconcile with the inversion of the higher gravitational moment $J_{4}$, made by Anderson, Hubbard, and Slattery (1974).

The fluid state of these planets is assured by showing that the adiabatic temperature profile which matches the deep atmosphere gives a temperature that exceeds the melting point of hydrogen (or the liquidus of a hydrogen-helium mixture) at each depth. To a very crude approximation, the Jovian adiabat is

$$
T \approx 10,000 \rho^{1 / 2} \mathrm{~K}
$$

where $\rho$ is in $\mathrm{g} \mathrm{cm}^{-3}$, and the Saturnian adiabat has the same form but is $10-20 \%$ colder. This temperature is comfortably in excess of the melting temperatures estimated in $\S$ II, Paper I. The fluid state ensures that convection is readily initiated once the adiabatic temperature is slightly exceeded, and is not inhibited by molecular viscosity.

To confirm the adiabatic hypothesis, it remains to be demonstrated that the thermal convection requires only a very small fractional superadiabaticity. Stevenson and Salpeter (1976) have discussed this for Jupiter, but almost identical numbers apply for Saturn. Even if allowance is made for the strongly inhibiting effect of rotation, the fractional superadiabaticity is found to be much smaller than unity. The effect of rotation has recently been analyzed in more detail (Gierasch and Stevenson 1977), and the same conclusion was reached. The inhibiting effect of the magnetic field is not expected to be greater than that of rotation, if a dynamo is operating, since the Lorentz force will be at most comparable to the Coriolis force (Hide 1974). Apparently, the only other conceivable inhibition of the convection is the molecular-metallic transition, but if this is continuous, then an element of fluid can change smoothly from one phase to the other as it moves through the pressure region of the transition. No supercooling or superheating would be possible, and a rising fluid element would always be only slightly less dense than the surrounding field. Of course, the region of the transition will in general have an "anomalously" large or "anomalously" small adiabatic temperature gradient. In the case where the adiabatic gradient is much larger in magnitude within the transition region than elsewhere, electronic conduction can become important and the adiabatic assumption could break down. This possibility is too unlikely to merit a discussion.

Provided there exist minor constituents to block the window in the molecular hydrogen opacity spectrum, the adiabatic approximation is valid for a homogeneous planet with no first-order phase transitions or immiscibilities.

Evolutionary calculations for Jupiter (Graboske et al. 1975; Hubbard 1977) and Saturn (Pollack et al. 1977) have been made only for this homogeneous, adiabatic case. The major part of the evolution is then 
the gradual loss of primordial heat during the degenerate cooling phase. To an adequate first approximation, the luminosity is then equal to the rate of change of internal thermal energy:

$$
L=4 \pi R^{2} \sigma\left(T_{e}^{4}-T_{0}^{4}\right) \approx-\frac{d}{d t}\left(\frac{4}{3} \pi R^{3} \bar{C}_{v} T_{i}\right),
$$

where $L$ is the excess luminosity, $R$ is the radius, $\sigma$ is the Stefan-Boltzmann constant, $T_{e}$ is the actual effective temperature, $T_{0}$ is the effective temperature in the absence of an internal heat source, $\bar{C}_{v}$ is the average specific heat per unit volume, and $T_{i}$ is some average internal temperature. Since the entire interior is assumed to be convective, $T_{i}$ is related to $T_{e}$ by being on the same adiabat:

$$
T_{i} \approx T_{e}\left(\frac{P_{i}}{P_{e}}\right)^{n}
$$

where $P_{i}$ is a characteristic internal pressure, $P_{e}$ is the effective pressure (i.e., the pressure at optical depth unity in the atmosphere) and $n \approx 0.25$ is the average adiabatic index. From the virial theorem (Clayton 1968),

$$
P_{i} \approx \frac{G M^{2}}{4 \pi R^{4}}
$$

while optical depth unity corresponds to

$$
P_{e} \approx \frac{g}{\kappa}
$$

where $g$ is the acceleration due to gravity and $\kappa$ is the effective transmission opacity of the atmosphere. In the degenerate cooling phase, $T_{i}$ changes more rapidly with time than $C_{v}$ or $R$. Furthermore, the atmospheric models of Graboske et al. (1975) indicate that $\kappa$ changes little, even as $T_{e}$ changes by an order of magnitude. It follows that $P_{i}$ and $P_{e}$ can be regarded as constant during most of the evolution, so that $T_{i} \propto T_{e}$. The solution of equation (2) is then

$$
\begin{aligned}
& t_{0}=\frac{(\alpha) \text { (present heat content) }}{\text { (present excess luminosity) }} \\
& \alpha \equiv\left(1-q^{4}\right) \int_{1}^{x_{m}} \frac{d x}{x^{3}-q^{4}}
\end{aligned}
$$

where $t_{0}$ is the "age" of the planet (the time that has elapsed since it first became degenerate), $q=T_{0} / T_{e, f}$ where $T_{e, f}$ is the present effective temperature, and $x_{m}=T_{e, f} / T_{e, i}$ where $T_{e, i}$ is the effective temperature at the beginning of the degenerate cooling. The value of $\alpha$ is insensitive to $x_{m}$ for $x_{m} \geqslant 3$. In the limit as $x_{m} \rightarrow \infty$,

$$
\alpha=\frac{1}{3}\left[1-\frac{4 q^{4}}{7}-\frac{12 q^{8}}{77}+O\left(q^{12}\right)\right] .
$$

For both Jupiter and Saturn at present, $q^{4} \approx 0.5$ and $\alpha \approx 0.25$. The value of $\alpha$ is substantially less than unity because the luminosity increases rapidly as one goes back in time. For "typical" adiabatic, homogeneous models of Jupiter (Stevenson and Salpeter 1976) and Saturn (Podolak 1974), one finds $t_{0} \approx 4 \times$ $10^{9}$ for Jupiter and $t_{0} \approx 2 \times 10^{9}$ years for Saturn, each with about $1 \times 10^{9}$ years' uncertainty. The more precise evolutionary calculations for Jupiter (Graboske et al. 1975; Hubbard 1977) and Saturn (Pollack et al. 1977) do not differ greatly from the above crude analysis. The major uncertainties are the present luminosity, the transmission opacity, the specific heat in the deep interior, and the average adiabatic gradient. The calculation suggests that a homogeneous Jupiter with no first-order phase transitions is consistent with the assumed age of about $4.5 \times 10^{9}$ years. (There is no direct evidence relating to the ages of the major planets, but neither is there any reason to believe that they differ greatly in age from the terrestrial planets.) The uncertainties (especially in the present luminosity) are greater for Saturn, but the small value of $t_{0}$ derived for that planet suggests that Saturn may not be homogeneous, or at least may have a different evolution from Jupiter. In "natural" (i.e., gravitational) units, Saturn has an "anomalously" large excess luminosity (see Stevenson 1977). The two most likely explanations are either that Saturn is inhomogeneous or that observers have overestimated the excess luminosity. This dilemma may be resolved with the flyby of Saturn by Pioneer 11 in 1979. In $\S$ IV and V, we examine the hypothesis that inhomogeneity is the explanation. We are not precluding inhomogeneity in Jupiter either, since the uncertainties are still large in the homogeneous evolution. Furthermore, even if the planets were pure hydrogen, the adiabatic assumption would not be valid if the molecular-metallic transition were firstorder. At the end of the next section we discuss how this can also affect the evolutionary time scale.

\section{THE MOLECULAR-METALLIC HYDROGEN TRANSITION}

We consider now a pure hydrogen planet in which the molecular-to-metallic hydrogen transition is firstorder at the temperatures of interest, but in which the conductivity is always low enough (or the opacity high enough) to ensure convection everywhere well away from the transition. In a recent paper, Salpeter and Stevenson (1976) consider a self-gravitating fluid, stratified into two phases of appreciably different densities and heated from within. It is assumed that, away from the interface between the phases, the heat flux is mainly carried by turbulent convection with a very small superadiabaticity. Different modes are investigated for transporting the heat flux across the interface, and both possible signs for the phasetransition latent heat $L$ are considered. Under a wide range of conditions, it is found that the transition region near the interface is thin, with a small change in temperature across it. The entropy difference between the two phases is then $L / T$, where $T$ is the temperature at the transition. In reaching this conclusion, the following assumptions were needed: (i) a fractional density change at the transition that is not enormously less than 
unity; (ii) a substantial positive surface energy $\sigma$ between the phases, at both microscopic and macroscopic levels; (iii) a substantial latent heat $L$, with magnitude of order $k_{B} T$ per particle, where $k_{B}$ is Boltzmann's constant; (iv) a heat flux which is determined by conditions elsewhere, and whose average is not affected by the dynamics of the phase transition (in the case of Jupiter and Saturn, the heat flux is determined by conditions in the surface layers of the planet and its central temperature); (v) a Prandtl number (defined as $P r \equiv \nu / \kappa$, where $\nu$ is the kinematic viscosity and $\kappa$ is the thermal diffusion coefficient) that is not so enormously greater than unity that large-scale convective flows are inhibited by viscosity.

Of all these conditions, (ii) and (v) are particularly crucial. If the molecular-metallic hydrogen transition is indeed first-order (see the discussion in Paper I), then these conditions are probably satisfied.

This conclusion is in contrast to that reached by Schubert, Turcotte, and Oxburgh (1970) in their discussion of the olivine-spinel solid-state phase transition in the Earth's mantle. They propose no entropy discontinuity, but rather a "two-phase" region where the two phases are intermingled and neither phase predominates. To understand why their conclusion is not incompatible with ours, two aspects of the problem must be considered: the predictions of linear stability analysis, and the nature of the finite amplitude flow.

A linear stability analysis was carried out for $L>0$ by Busse and Schubert (1971). They found that a state in which the phases are stratified with a welldefined interface becomes unstable to mixing when the superadiabaticity becomes so large that an upwardmoving parcel of fluid can change phase, cool down (because of the latent heat), and yet still remain buoyant. For $L \approx k_{B} T$, this requires a fractional superadiabaticity of order unity. This instability criterion is apparently satisfied in the Earth, where viscosity greatly inhibits the flow in the solid phases, and the superadiabaticity must be large. This criterion is not satisfied for fluid phases in Jupiter or Saturn, where the superadiabaticity has a very small average value.

The second aspect of the problem is the nature of the finite amplitude flow. Turcotte and Schubert (1971) consider a simple, one-dimensional model for the flow and deduce a "two-phase" region. Since the two phases have different densities, there is a tendency for them to separate under the action of gravity. However, in the high viscosities prevailing in the Earth's mantle, the rate of separation is no greater than convective speeds elsewhere, so a dynamic steady state can be envisaged in which a two-phase region persists. In our situation, where molecular viscosity is essentially irrelevant, no two-phase region is conceivable in steady state, since it would separate almost at sound speed, on a time scale much less than typical convective time scales. To summarize, the most important difference between the Earth's mantle and the interiors of fluid hydrogen-helium planets is the factor of $\sim 10^{24}$ difference in Prandtl numbers.

This does not prove that our conclusion of an essentially "isothermal" (rather than "adiabatic") interface is correct. To prove that, we would need to consider all possible modes for finite-amplitude disturbance of the interface. This has not been done, but those modes that were considered were found to be stable (Salpeter and Stevenson 1976). Turner (private communication) has pointed out that a major (possibly the major) source of mass transfer between the phases was not considered in Salpeter and Stevenson (1976). Experiments on turbulent entrainment across density interfaces (between fluids of different composition) in the large Reynold's number limit (Turner 1968b; Linden 1973; Long 1975) indicate that a small amount is ejected at high speed from one fluid into the other during the recoil of a large eddy that has hit the interface. The ejection velocity is comparable to $\mathscr{U}_{W}$, the wave velocity on the interface:

$$
\mathscr{U}_{W}=\left(g l \frac{\Delta \rho}{\rho}\right)^{1 / 2},
$$

where $g$ is the acceleration due to gravity, $l$ is a length scale characterizing the turbulence (i.e., eddy size), $\Delta \rho$ is the density contrast at the interface, and $\rho$ is the average fluid density. The amount ejected (in each direction) can be expressed as an entrainment velocity $\mathscr{U}_{e}$ (the ejected volume per unit interface area per unit time) given by

$$
\frac{\mathscr{U}_{e}}{\mathscr{U}}=\left(\frac{\mathscr{U}}{\mathscr{U}_{\mathrm{W}}}\right)^{n},
$$

where $\mathscr{U}$ is a characteristic turbulent (convective) velocity for eddy size $l$, and $n=3$ according to Turner (1968b) and Linden (1973). Neglecting rotation, $\mathscr{U} \approx 10 \mathrm{~cm} \mathrm{~s}^{-1}$ for Jupiter and Saturn and $l \approx 10^{9} \mathrm{~cm}$ (Hubbard and Smoluchowski 1973), so that $\mathscr{U}_{e} \approx$ $10^{-14} \mathrm{~cm} \mathrm{~s}^{-1}$. The latent heat flux $\mathscr{U}_{e} L$ is therefore $\leq 10^{-2} \mathrm{ergs} \mathrm{cm}^{-2} \mathrm{~s}^{-1}$ in magnitude, and negligible compared with the sensible heat flux. Unlike the experiments, the two fluids are phases of the same substance and the net effect of ejection is zero. (There is, however, a small but finite probability of encountering a macroscopic amount of the "wrong" phase at large distances from the phase boundary.)

Experiments by Long on shear-induced turbulence (1975) have been interpreted as implying $n=2$. In this case, both the latent heat and sensible heat fluxes are proportional to $\mathscr{U}^{3}$, but the latent heat flux is nevertheless smaller by $|L| / \rho \mathscr{U}_{W}{ }^{2}<1$. In this case, the entrained fluid, although small in total volume, can have a thermal effect comparable to the sensible heat flux. Even if Long's experiments are applicable (which they probably are not), the interface would still be well defined, although the convection would be substantially different from the "normal" $(n=3)$ case.

An "isothermal" interface appears to be ensured provided $\mathscr{U} \ll \mathscr{U}_{\mathrm{W}}$ and $R_{e} \equiv \mathscr{U} l / \nu \gg 1$, where $\nu$ is the kinematic viscosity. The conclusions of Salpeter and Stevenson (1976) can be applied to Jupiter and Saturn as follows: In the molecular-metallic hydrogen transition, the metallic phase is about $30 \%$ more dense than the molecular phase. The sign of $L$ is not known, but 


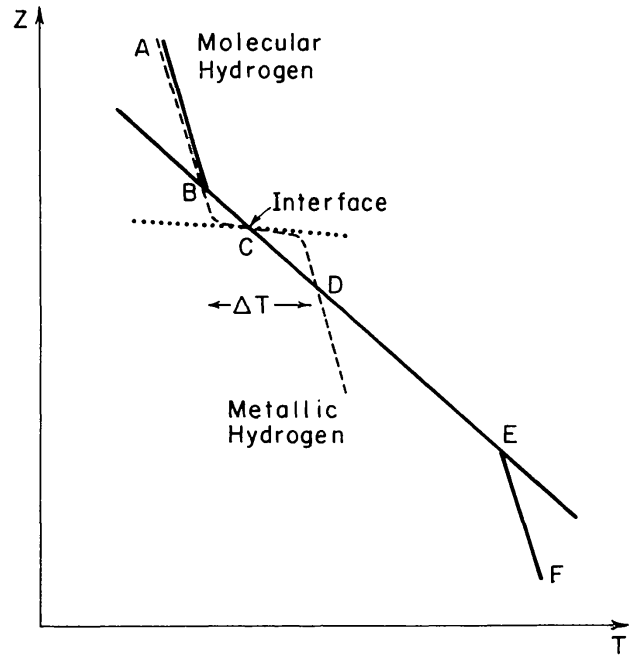

Fig. 2.-Temperature versus vertical coordinate $z$, for positive latent heat and no nucleation. $B C D E$ is part of the phase boundary, while $A B$ and $E F$ are adiabats corresponding to the same specific entropy. In a fully adiabatic case, the temperature profile would be $A B E F$, with a two-phase region between $B$ and $E$. The actual temperature profile (---) is almost adiabatic except for a thin region near the interface. This region, labeled by $\Delta T$, is exaggerated for clarity. The temperature profile for pure conduction $(\cdots)$ is also shown.

$|L| / k_{B} T$ is probably slightly less than unity (Stevenson and Salpeter 1976). Consider the case where $L>0$ and no nucleation of one phase within the bulk of the other is possible. We predict the formation of a thermal boundary layer between the phases, in which heat conduction dominates (small-scale convection is inhibited by heat leakage or molecular viscosity). A simple mixing-length analysis yields a boundary layer thickness of order $10 \mathrm{~cm}$, across which there is a very small temperature drop $\Delta T \approx 10^{-2} \mathrm{~K}$, as shown in Figure 2 ( $\Delta T$ is enlarged for clarity). Flow across the phase boundary is inhibited by the density difference and the inability of a macroscopic volume of fluid to change phase instantaneously. Instead, there are gravity waves on the interface, with amplitudes as great as $10^{3} \mathrm{~cm}$ for the longest wavelengths $\lambda \approx 10^{9}$ $\mathrm{cm}$. This mainly represents a moving up and down of the boundary layer, with the actual thickness of the boundary layer itself being appreciably less.

Suppose, now, that nucleation is possible. It is evident from Figure 2 that the fluid between $B$ and the interface $C$ is supercooled and molecular, while the fluid between $C$ and $D$ is superheated and metallic. At $T \approx 10^{4} \mathrm{~K}$ in Jupiter or Saturn, homogeneous nucleation is probably the only nucleation mechanism. Using a surface energy comparable to that of pure metallic hydrogen relative to vacuum (about $0.1 \mathrm{eV}$ per surface atom, according to the theory of Lang and Kohn 1970), Salpeter and Stevenson find that the amount of superheating or supercooling is never enough to initiate significant nucleation. If heterogeneous nucleation were somehow possible, then only infinitesimal superheating or supercooling might be needed. However, it is still not possible for a large amount of fluid to rapidly change phase, since the superheating (or supercooling) is generally much less than the latent heat. Consider, for example, a crest of metallic hydrogen on the wavy interface. Since the interface itself can be neither superheated nor supercooled, the interface itself lies on the phase boundary. However, the fluid just below the crest is superheated and metallic. If nucleation seeds are available, then bubbles of the molecular phase begin to grow at a rate determined by the diffusion of heat onto the bubble. However, only a small amount of fluid has changed phase before the entire crest has cooled to the local phase boundary, and superheating no longer exists. This nucleation process cools the metallic hydrogen and thus contributes to an upward heat flux. Since the total heat flux must be constant, it follows that the thermal profile will rearrange itself so that the interface is actually more hydrodynamically quiescent than it would be in the absence of nucleation.

In the case $L<0$, no supercooled or superheated regions arise, and the thermal boundary layer is similar to that for $L>0$ if there are no waves at the interface. The phase change of fluid at the interface in a wave crest or trough might enhance the upward heat flux, so a temperature inversion may be needed to inhibit excessive heat flow. This temperature inversion is at most about $\Delta T \approx 10^{-3} T \approx 10 \mathrm{~K}$.

The effect of planetary rotation on these considerations is small. Far from the interface, the superadiabaticity is much larger in the presence of rotation than in its absence, but it is still much less than unity. Simple mixing-length theory (without rotation) predicts a fractional superadiabaticity $\epsilon \approx 10^{-8}$ in Jupiter or Saturn, if the mixing length is of the order of the pressure scale height. Allowance for rotation (Stevenson and Salpeter 1976; Gierasch and Stevenson 1977) yields $\epsilon \approx 10^{-4}$, in similar circumstances. As one approaches the interface, a point is reached at which rotation is no longer important (i.e., Coriolis force becomes smaller than buoyancy force). This occurs at a distance $z$ from the interface, given by

$$
\frac{v(z)}{z \Omega} \approx 1
$$

where $v(z)$ is the convective velocity appropriate to a mixing length $z$, and $\Omega$ is the planetary angular velocity. This is satisfied in Jupiter or Saturn by $z \approx 10^{5} \mathrm{~cm}$, within an order of magnitude. Since the thermal boundary layer is much thinner than this, rotation is not rapid enough to change its structure.

The effect of magnetic fields on the structure of the interface is difficult to assess, especially if there is a large discontinuity in electrical properties across the interface. According to most dynamo theories (Stevenson 1974) the Lorentz force is no greater than the Coriolis force, so it seems likely that magnetic field effects are unimportant, if rotation is unimportant. Magnetic "buoyancy" of the metallic fluid immediately below the interface may enhance the amplitude of interfacial waves, but since magnetic pressure is probably many orders of magnitude less than the 
hydrostatic pressure, this should not be an important consideration.

To summarize: If the molecular-to-metallic transition is first-order, and the conclusions of Salpeter and Stevenson (1976) are applicable, then large deviations from full adiabaticity may result. In contrast to Hubbard's hypothesis, which states that

$$
S_{c}=S_{\mathrm{atm}},
$$

where $S_{c}, S_{\mathrm{atm}}$ are the specific entropies of the central and atmospheric regions of the planet, respectively, we have instead

$$
S_{c}+\Delta S=S_{\text {atm }},
$$

where $\Delta S=L / T$ is the entropy change at the transition. It follows that a central temperature $T_{c}$ evaluated according to equation (11) could be wrong by as much as a factor of 2 (Stevenson and Salpeter 1976) in either sense. This is an extreme upper bound, and it is more likely that $T_{c}$ determined by equation (11) is wrong by only $10 \%$ or $20 \%$, but even this is not negligible in an accurate interior model. (The uncertainty in $\Delta S$ is essentially the uncertainty in the adiabat for molecular hydrogen at $\rho \geqslant 0.1 \mathrm{~g} \mathrm{~cm}^{-3}$, since the adiabats are well known at lower densities and at metallic densities. All models of Jupiter and Saturn-except Stevenson and Salpeter [1976] -implicitly assume $\Delta S=0$.)

The existence or absence of a well-defined interface is a qualitative feature which may have observable consequences for the multipolarity of the magnetic field, the large-scale convective pattern (Busse 1976), or the normal modes of the planet, in addition to modifying the compositional and thermal structure.

We consider now the effect of this first-order phase transition on the cooling of the planet. For simplicity, we assume that the actual temperature at the phase boundary is much less than the critical temperature for the first-order character of the transition, and we assume that the entropy change and volume change at the transition are independent of temperature. There are two ways in which the cooling rate differs from that for an adiabatic, homogeneous planet. First, the present heat content is different since the specific entropy in the metallic core is no longer equal to the specific entropy in the atmosphere (eq. [12]). This is a primordial latent heat effect (i.e., the nonadiabatic structure resulted during the formation or very early evolution of the planet). Second, the phase boundary is evolving as the planet cools, because of the temperature-dependence of the transition pressure. This is a contemporary latent heat effect.

The primordial latent heat effect is readily evaluated by noting that the age of the planet is proportional to its present heat content (eq. [6]), provided the planet is homogeneous. In Jupiter, most of the present heat content is in the metallic core, and the temperature in this core differs from that for an adiabatic homogeneous planet by a multiplicative factor $\exp (-\Delta S / 2)$, where $\Delta S$ is the entropy change at the transition in $k_{B}$ per proton (Stevenson and Salpeter 1976). The age of the planet is therefore modified by roughly the same multiplicative factor. This factor could be as small as 0.5 or as large as 2.0 , but is probably closer to unity. The effect on Saturn is smaller, since a smaller fraction of the total heat content resides in the metallic core or in very dense molecular hydrogen.

The contemporary latent heat effect is much smaller. As the planet cools, one phase grows at the expense of the other. This leads to gravitational and internal energy changes that almost compensate, the net effect being the purely thermal one of latent heat release (Flasar 1973). According to the Clausius-Clapeyron equation,

$$
\left(\frac{d P}{d T}\right)_{\mathrm{ph}}=\frac{\Delta S}{\Delta v}
$$

where the derivative is evaluated along the phase boundary, and $\Delta v \approx 3 a_{0}{ }^{3} /$ proton (Stevenson and Salpeter 1976) is the volume change at the transition. The additional luminosity from latent heat generated at the boundary, $Q_{L}$, is

$$
Q_{L} \approx-\frac{4 \pi R^{2} L}{g}\left(\frac{d P}{d T}\right)_{\mathrm{ph}}\left(\frac{d T}{d t}\right),
$$

where $L=T \Delta S$ is the latent heat per gram, and $(d T / d t)$ is the rate at which the temperature is changing at the phase boundary. Assuming $d T / d t \approx-2 \times$ $10^{-14} \mathrm{~K} \mathrm{~s}^{-1}$, which is appropriate to adiabatic, homogeneous models of Jupiter (see $\S$ II), one finds that for $T \approx 10^{4} \mathrm{~K}$,

$$
Q_{L} \approx 6 \times 10^{23}(\Delta S)^{2} \mathrm{ergs} \mathrm{s}^{-1},
$$

where $\Delta S$ is in $k_{B}$ per proton. Since $|\Delta S|<1 k_{B}$ per proton (Stevenson and Salpeter 1976), it follows that $Q_{L}$ is at most $10 \%$ of the total heat flux of $5 \times 10^{24}$ ergs $\mathrm{s}^{-1}$. In Saturn, the inequality is even greater because of the smallness of the metallic core. Note that $Q_{L}$ is positive regardless of the sign of $\Delta S$. (If $\Delta S>0$, then the metallic core grows at the expense of the molecular mantle. If $\Delta S<0$, then the molecular mantle grows at the expense of the metallic core. In either case, heat is released.)

These calculations are of limited usefulness for Jupiter and Saturn, which are not pure hydrogen. In fact, both planets contain a substantial mass fraction of helium. The Gibbs phase rule enforces a discontinuity of helium fraction at a first-order molecularmetallic phase transition, and this can have a much larger effect on the cooling rate (see $\S \mathrm{VI}$ ).

\section{CONVECTION IN THE PRESENCE OF A COMPOSITIONAL GRADIENT}

Thermal convection in the presence of composition gradients is not a simple generalization of homogeneous thermal convection, because the additional available degrees of freedom can admit qualitatively new phenomena. There is an extensive literature on this problem (see, for example, Spiegel 1972), but we limit ourselves here to those conditions which arise in hydrogenhelium planets when the helium is nonuniformly 
distributed. In particular, we assume that $D<\kappa$ always, where $D$ is the helium diffusivity and $\kappa$ is the thermal diffusivity. We also assume that the temperature gradient is destabilizing. The first assumption is almost certainly valid for both molecular and metallic phases (see Paper I, $\S$ VII and VIII).

With these assumptions, it is possible to eliminate the "salt finger" modes (Turner 1967). The remaining steady states are: purely diffusive, overstable, and unstable. The purely diffusive solution is well understood and exactly solvable. It need not concern us further. The unstable mode is a simple generalization of homogeneous thermal convection, and is highly efficient in the transport of heat or solute. The overstable mode is qualitatively new and owes its existence to the presence of two diffusive processes of different efficiencies (Shirtcliffe 1967; Turner 1968a).

Consider, first, the unstable mode. In direct analogy to the well-known simple mixing-length theory, we can consider a parcel of fluid in equilibrium with the ambient medium, with composition and density given by $x$ and $\rho$, respectively. The parcel is then displaced upward, expanding adiabatically and maintaining the same composition. The condition for instability is that the parcel must then have lower density than the ambient fluid, i.e.,

$$
\left(\frac{\partial \rho}{\partial p}\right)_{x, s}<\frac{d \rho}{d p}
$$

where $s$ is the entropy, $p$ is the pressure, and

$$
\frac{d \rho}{d p}=\left(\frac{\partial \rho}{\partial p}\right)_{x, s}+\left(\frac{\partial \rho}{\partial x}\right)_{s, p}\left(\frac{d x}{d p}\right)+\left(\frac{\partial \rho}{\partial s}\right)_{x, p}\left(\frac{d s}{d p}\right),
$$

which, after some elementary manipulation, becomes

$$
\begin{aligned}
\frac{d \rho}{d p}= & \left(\frac{\partial \rho}{\partial p}\right)_{x, s}+\left(\frac{\partial \rho}{\partial x}\right)_{p, T}\left(\frac{d x}{d p}\right) \\
& +\left(\frac{\partial \rho}{\partial T}\right)_{x, p}\left[\frac{d T}{d p}-\left(\frac{\partial T}{\partial p}\right)_{x, s}\right] .
\end{aligned}
$$

If we define

$$
\begin{aligned}
& \epsilon=\frac{1}{\rho}\left(\frac{\partial \rho}{\partial T}\right)_{x, p}\left[\frac{d T}{d p}-\left(\frac{\partial T}{\partial p}\right)_{x, s}\right] \frac{d p}{d z} H_{p}, \\
& \chi=-\frac{1}{\rho}\left(\frac{\partial \rho}{\partial x}\right)_{q, T} \frac{d x}{d z} H_{p},
\end{aligned}
$$

where $z$ is a vertical coordinate and $H_{p}$ is the pressure scale-height, then

$$
\epsilon>\chi
$$

is the condition for instability. Generalizing the usual arguments of simple mixing-length theory, we can then derive a velocity $v$ :

$$
v \approx v_{s}(\epsilon-\chi)^{1 / 2}\left(l / H_{p}\right),
$$

where $l$ is the mixing length, $v_{s}=\left(g H_{p}\right)^{1 / 2}$ is the sound speed, and $g$ is the acceleration due to gravity. The heat flux $F_{T}$ is of order

$$
F_{T} \approx \gamma \rho v_{s}^{3} \epsilon(\epsilon-\chi)^{1 / 2}\left(l / H_{p}\right)^{2},
$$

where we have used the fact that

$$
\begin{gathered}
\frac{C_{p} T}{\alpha}=\gamma \frac{p}{\rho} \approx \gamma v_{s}{ }^{2}, \\
\alpha=-\left(\frac{\partial \ln \rho}{\partial \ln T}\right)_{q, x}, \quad \gamma=\left(\frac{\partial \ln P}{\partial \ln T}\right)_{s, x},
\end{gathered}
$$

and $C_{p}$ is the constant pressure specific heat. We can also evaluate the solute mass flux $F_{x}$ :

$$
F_{x} \approx \rho v_{s} \chi(\epsilon-\chi)^{1 / 2}\left(l / H_{p}\right)^{2} .
$$

The rate at which work is done against gravity in redistributing the solute is of order $v_{s}^{2} F_{x}\left(l / H_{p}\right)$. An obvious consequence of these results is that a very small compositional gradient can have a large effect on the convection properties. For example, $\epsilon \approx 10^{-8}$ in Jupiter if $\chi \equiv 0$, and the effect of rotation is neglected (as it is above). Thus, if $\chi \geqslant 10^{-8}$, the convection properties would be modified. In the next section, we consider situations in which $\chi \approx 1$. The effect of rotation is not negligible, of course, but it does not change the instability criterion, and roughly speaking just changes the right sides of equations (20) and (22) by the same multiplicative factor $\delta(l)$. [For Jupiter, $\delta\left(H_{p}\right) \approx 10^{-6}$, so that $\epsilon \approx 10^{-4}$ for $\chi \equiv 0, l=H_{p}$ (Gierasch and Stevenson 1977).]

Consider now the overstable mode. In this mode, the fluid is stably stratified $(\epsilon<\chi)$, but small-scale fluid oscillations can grow because of the greater efficiency of heat diffusion relative to helium diffusion. Consider a displacement of an element of fluid that is sufficiently small for molecular diffusion effects to be significant. In the displaced position, heat and solute diffuse from the fluid element into the surrounding ambient medium. If the density increase from this heat diffusion exceeds the density decrease from the solute diffusion, then the density contrast between the fluid element and the ambient medium is enhanced, and a growing oscillation is possible, driven by the thermal buoyancy force. In the absence of viscosity, the condition for overstability is

$$
\kappa \epsilon>D \chi .
$$

Molecular viscosity $\nu$ is always important, however, and the correct result incorporating $\nu$ is (Walin 1964)

$$
(\kappa+\nu) \epsilon>(D+\nu) \chi
$$

for overstability. The regime of overstability is slice of $(\epsilon, \chi)$-space, bounded on one side $(\epsilon>\chi)$ by the unstable region and on the other side by the stable (diffusive) regime. In Figure 3, the stability diagram is given for the situation of interest $(\kappa>D \approx \nu$, $\epsilon>0, \chi>0$ ).

The overstable mode is most efficient when the characteristic time for heat diffusion across a fluid 


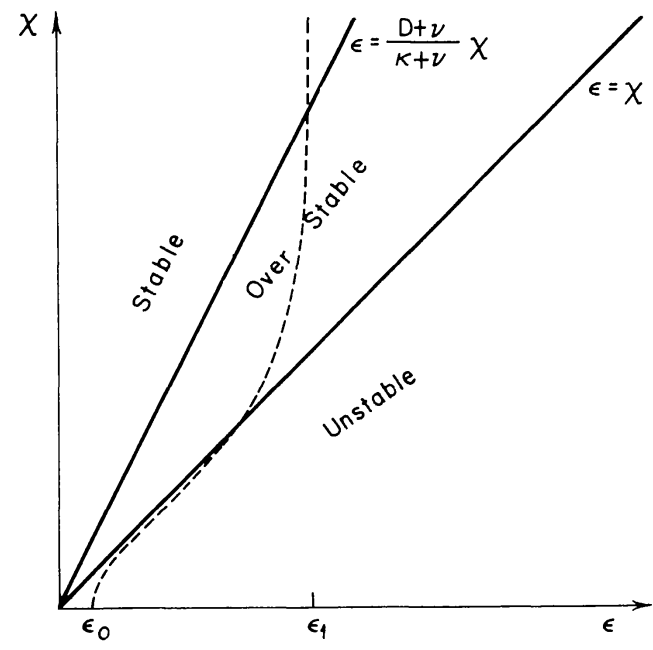

FIG. 3.-The stability diagram for thermosolutal convection, assuming $\epsilon>0, \chi>0, \kappa>D$. The dashed line schematically represents a constant heat flux contour. For clarity, the $\chi=0$ intercept $\left(\epsilon=\epsilon_{0}\right)$ is shown well-displaced from the origin. Usually $\epsilon_{1}$ (the value of $\epsilon$ for pure heat conduction) is many orders of magnitude larger than $\epsilon_{0}$. The transition from unstability to overstability (at a given heat flux) is not well defined, but occurs in a region of $\epsilon$ that is not greatly less than $\epsilon_{1}$.

element is comparable to the oscillation time:

$$
\lambda^{2} / \kappa \approx\left(H_{p} / v_{s}\right)(\chi-\epsilon)^{-1 / 2},
$$

provided $\nu$ is not many orders of magnitude greater than $\kappa$. The characteristic horizontal length scale ("wavelength") $\lambda$ is typically of order $10 \mathrm{~cm}$ in the situations of interest $(\chi-\epsilon \approx 1)$. The vertical amplitude cannot be estimated from linear stability analysis, but experiments (Caldwell 1974) indicate that heat and solute fluxes are not very much greater than they would be from pure diffusion. This means that the amplitude of the oscillations is never enormously greater than the wavelength, a physically reasonable conclusion. Overstability should therefore be regarded as a mechanically enhanced diffusion process rather than a convective mixing process. This means that the ratio of thermal to solute fluxes should be roughly the same as it would be if only diffusion were acting. (This is only true for $\epsilon \geqslant \alpha$ since thermal diffusion is driven by the total temperature gradient, not just the superadiabatic excess. This criterion is always satisfied in laboratory-sized experiments, and is satisfied in many of the situations that we consider in subsequent sections.)

In Figure 3, the dashed line schematically indicates a contour of constant heat flux. In the stable region, $\epsilon=\epsilon_{1}$ (a constant for all $\chi$ if we neglect the Soret effect-see Paper I, $\S$ VII). The onset of overstability is accompanied by a gradual reduction in $\epsilon$ for a given heat flux, but because of the inherent inefficiency of the overstable modes relative to normal convection, the reduction in $\epsilon$ is never very great, probably less than an order of magnitude. The transition from overstable to unstable behavior is complicated, and is not accurately represented in Figure 3. Once unstability predominates, equation (20) shows that $\epsilon-\chi \ll \epsilon$ until near $\chi=0$, where $\epsilon \approx \epsilon_{0}+\chi / 3$. An interesting feature of the unstable regime in which $\epsilon-\chi \ll \epsilon$ is that equation (19) then predicts very slow convective velocities. Under these circumstances, convection is likely to be intermittent.

In thermosolutal convection, nonlocal (Turner and Stommel 1964) and time-dependent effects may occur. The following situation is of particular relevance in evolving hydrogen-helium planets.

Consider a semi-infinite pure fluid, bounded below by a rigid, perfectly conducting plate. Incident on this plate is a constant, given upward heat flux $F_{T}$. Experiments and theory (Howard 1964) indicate that an intermittent boundary layer is formed which grows by thermal diffusion until the local Rayleigh number is exceeded for a layer of thickness $\sim(\kappa t)^{1 / 2}$, where $t$ is the elapsed time and $\kappa$ is the thermal diffusivity. A thermal plume forms which removes the buoyant fluid from the plate, and the whole process is then repeated. Now suppose that solute is also introduced at the plane $z=0$ at a constant mass rate $F_{x}$. Assume that at $t=0$ there is no deviation from neutral stability in the fluid, and let $\Delta \rho_{T}$ and $\Delta \rho_{x}$ be the subsequent $z=0$ density changes caused by heat and solute. (Both are defined to be positive, but the thermal effect is destabilizing and the solute effect is stabilizing.) The exact form of the subsequent diffusive solution need not concern us (see, for example, Jeffreys and Jeffreys 1950), but the general features are that $(a)$ both $\Delta \rho_{T}$ and $\Delta \sigma_{x}$ increase as $t^{1 / 2}$ and their ratio is constant; $(b)$ the characteristic distances over which the density changes extend are $(\kappa t)^{1 / 2}$ and $(D t)^{1 / 2}$ for heat and solute, respectively. Let $F_{T}{ }^{*}$ and $F_{x}{ }^{*}$ be the respective $z=0$ fluxes in density units. It follows that

$$
F_{T} * \approx \frac{\kappa \Delta \rho_{T}}{(\kappa t)^{1 / 2}}, \quad F_{x}^{*} \approx \frac{D \Delta \rho_{x}}{(D t)^{1 / 2}} .
$$

These equations are approximate, but the ratio equation is exact:

$$
\frac{F_{x}{ }^{*}}{F_{T}{ }^{*}}=\left(\frac{D}{\kappa}\right)^{1 / 2} \frac{\Delta \rho_{x}}{\Delta \rho_{T}}
$$

Provided $\Delta \rho_{T}>\Delta \rho_{x}$, a thermal can still form at the plate surface, and all the introduced solute can be transported away by convection. However, if $\Delta \rho_{x}>$ $\Delta \rho_{T}$, then a stable layer must form near $z=0$. Experiment and theory (Linden 1974; Linden and Shirtcliffe 1976) show that a diffusive "core" forms. At the edge of this core there is a new intermittent boundary layer which has the property that $F_{x}{ }^{*}=(D / \kappa)^{1 / 2} F_{T}{ }^{*}$ locally. To conclude: If $F_{x}{ }^{*} \leq(D / \kappa)^{1 / 2} F_{T}{ }^{*}$ at $z=0$, then all the introduced solute can be transported away by convection. If $F_{x}{ }^{*}>(D / \kappa)^{1 / 2} F_{T}{ }^{*}$, then a stable diffusive layer grows, and the amount of solute transported away by convection is at most $(D / \kappa)^{1 / 2} F_{T}{ }^{*}$ in density units. For relevant values of $D$ and $\kappa$ (see Paper I) this limits the work done in redistributing helium upward to $\sim 10 \%$ of the thermal energy flux. This limit applies to initially localized perturbations of the helium frac- 
tion (e.g., at an interface between phases, or an interface between convective and diffusive or overstable regions).

In addition to the diffusive-convective equilibrium described here, there is direct mixing of helium by entrainment (i.e., wave-breaking at the interface). This is negligible if convective speeds are more than an order of magnitude smaller than wave speeds (Linden 1974). This criterion is satisfied in most cases.

Finally, we should consider whether more complicated global instabilities are favored relative to the simple steady states already considered. A common situation in experiments (Turner and Stommel 1964) is the formation of a steplike distribution of solute, in which uniformly mixed convective layers are separated by thin, diffusive layers where the temperature and solute concentration change rapidly. Experiment and theory (Linden and Shirtcliffe 1976) show that this is a possible steady state provided

$$
\frac{\Delta \rho_{x}}{\Delta \rho_{T}}<\left(\frac{\kappa}{D}\right)^{1 / 2},
$$

where $\Delta \rho_{T}, \Delta \rho_{x}$ (both positive) are now the total density drops across the fluid for the (destabilizing) superadiabatic temperature difference and (stabilizing) solute concentration difference, respectively. If this criterion is not satisfied, then the diffusive interfaces thicken with time and the system reverts to a purely diffusive or overstable state. Equation (28) may not be satisfied in some of the situations considered in subsequent sections. Furthermore, it is not clear whether layers could form at all. The usual laboratory and oceanographic situations in which layers form are not analogous to the planetary evolutions we consider in this paper.

\section{HELIUM IMMISCIBILITY}

In this section, we consider the effects of helium insolubility in a cooling hydrogen-helium planet. We assume throughout this section that the molecularmetallic hydrogen transition is not first-order. Nevertheless, the discussion of this section essentially corresponds to the "hot" case of Sector I in Figure 1.

The thermal energy content of Jupiter is about $3 \times 10^{42}$ ergs at present. An even larger energy is available, in principle, if Jupiter changed from a chemically homogeneous structure to one where the denser helium resides in a central core (Kiefer 1967; Flasar 1973). Helium differentiation was originally invoked to explain the excess luminosity of Jupiter (Smoluchowski 1967), but appears to be even more desirable for Saturn (Pollack et al. 1977).

It might be supposed that chemical separation and gravitational layering are impossible in the presence of fully developed turbulent convection, because diffusion times are enormously large compared with convective times. Salpeter (1973) pointed out that layering may nevertheless take place in the presence of convection, if helium becomes insoluble in hydrogen.

Salpeter originally proposed that this insolubility
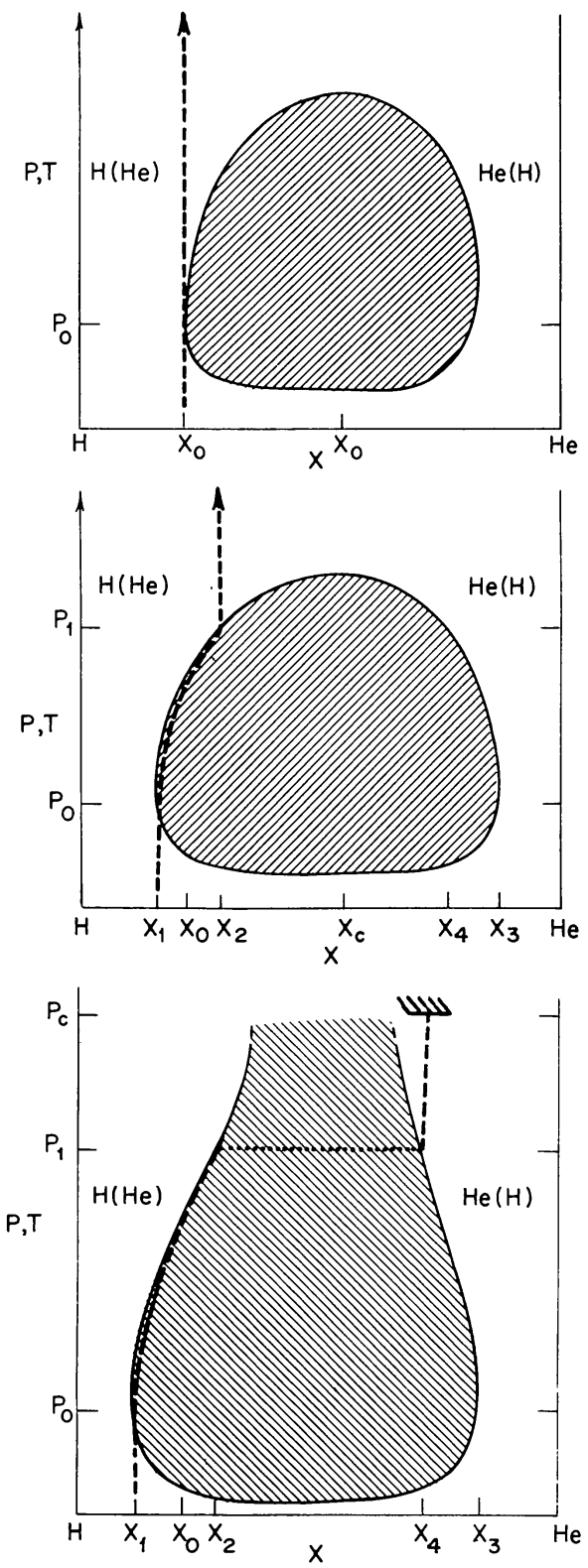

FIG. 4.-The inhomogeneous evolution of a hydrogenhelium planet in which the only first-order transition is helium immiscibility. The dashed line is the actual helium number fraction as a function of the actual pressure (or, equivalently, the actual temperature) within the planet. The region of immiscibility is shaded. The center of the planet (or the surface of a small rocky core) is $P=P_{c}$. In (a) (top), the planet is homogeneous, but phase separation is about to begin at $P=P_{0}$. In $(b)$ (middle), the planet has cooled down more, and the region of immiscibility has expanded somewhat. An inhomogeneous layer forms, but the helium-enriched central region is still predominantly hydrogen. In $(c)$ (bottom), the planet is cooler still, and now the inner region is predominantly helium.

would occur first in the metallic phase, but near the molecular-metallic transition. Our discussion in Paper I corroborates this guess. At the molecular-metallic transition, helium mixed in solar proportions first becomes insoluble when the temperature drops below about $8000 \mathrm{~K}$ (see Fig. 3, Paper I). The critical helium 
concentration $x_{c}$ substantially exceeds the primordial solar abundance $x_{0} \approx 0.1$ (Cameron 1973) where $x$ is the helium number fraction. A supercooled mixture of primordial composition would therefore preferentially separate into hydrogen-rich and helium-rich phases.

Suppose $T(P)$ is the actual temperature within the planet, $x(P)$ is the helium abundance, and $T_{\mathrm{ph}}(x, P)$ is the phase boundary temperature (the temperature below which the fluid would preferentially phaseseparate). At an early stage in the degenerate cooling phase of the planet, $T(P)>T_{\mathrm{ph}}\left(x_{0}, P\right)$ and $x(P)=x_{0}$ everywhere. Eventually, as the planet cools down, a time will be reached at which $T\left(P_{0}\right)=T_{\mathrm{ph}}\left(x_{0}, P_{0}\right)$ for some pressure $P_{0}$, close to the molecular-metallic transition, as shown in Figure $4 a$. A slight further reduction in temperature leads to a macroscopic layer of supercooled metastable fluid. Droplets of heliumrich fluid begin to nucleate from the mixture and grow. We consider three important questions: What size droplets are needed for efficient helium separation? Can droplets of this size be grown? How much supercooling is needed?

First, we consider how large a helium-rich droplet must be to have a terminal velocity in excess of typical convective speeds $\left(\sim 10 \mathrm{~cm} \mathrm{~s}^{-1}\right)$. This convective speed is derivable from mixing-length theory (with the effects of planetary rotation incorporated [Gierasch and Stevenson 1977]). Let $b$ be the radius of a droplet, let $V_{b}$ be its terminal velocity, and let $\Delta \rho$ be the density difference between the helium droplet and the surrounding fluid. The velocity is found by equating gravitational and drag forces:

$$
C_{D} \rho V_{b}^{2} b^{2} \approx \Delta \rho b^{3} g,
$$

where $C_{D}$ is the drag coefficient. Assuming $\mathrm{Re} \equiv$ $b V_{b} / \nu \geqslant 10^{3}$, we can approximate $C_{D} \approx 0.05$ (Landau and Lifshitz 1959). It is also adequate to approximate $\Delta \rho \approx \rho$. Thus

$$
V_{b}^{2} \approx 20 b g,
$$

and $V_{b} \geqslant 10 \mathrm{~cm} \mathrm{~s}^{-1}$ provided $b \geqslant 1 \mathrm{~cm}$. For $b \approx$ $1 \mathrm{~cm}, \operatorname{Re} \approx 10^{4}$, confirming our choice of $C_{D}$.

The diffusivity of helium in metallic hydrogen is roughly $D \approx 10^{-3} \mathrm{~cm}^{2} \mathrm{~s}^{-1}$ (Paper I), so the characteristic diffusion time for the droplet is $b^{2} / D \approx 10^{3} \mathrm{~s}$. This time is much less than $10^{8} \mathrm{~s}$, a typical large-scale convective time scale, so droplets can grow large enough to overcome convective motion before they are transported by convection to a region where they would preferentially dissolve. However, we must also consider whether droplets of this size are fragmented by the hydrodynamic pressure differences on the droplet surface. A measure of the distortion of the droplet from a sphere is the ratio of the work done by the hydrodynamic pressure in distorting a droplet to the additional surface energy created. This ratio is $\delta$, where

$$
\delta=\left(\frac{P a_{0}^{3}}{\sigma}\right)\left(\frac{b}{a_{0}}\right)\left(\frac{v_{b}}{v_{s}}\right)^{2},
$$

$a_{0}$ is a typical interparticle separation, $\sigma \approx 10^{-2} \mathrm{Ry}$ is the surface energy per surface particle, and $v_{s}$ is the sound velocity. For $b \approx 1 \mathrm{~cm}$, we find $\delta \approx 1$, so these droplets are near the maximum stable size. Regardless of the exact values of the parameters, it is clear that the downward flow of helium droplets is not highly inefficient.

Since the efficiency is not much less than unity, the gravitational energy release is at least of order $\rho b^{3} g J H$, where $J$ is the nucleation rate of droplets per unit surface area for the entire supercooled layer, and $H$ is the typical distance a droplet falls. The energy release could be much larger because each droplet can produce a cascade of droplets by successive fragmentations, but an upper bound to the nucleation rate (and the supercooling) can be found by ignoring this complication. The homogeneous nucleation rate is given by Feder et al. (1966) as

$$
J=\frac{H v_{s}}{a_{0}{ }^{4}} \exp \left[\frac{-\sigma^{3} k_{B} T}{2 \lambda^{2}\left(k_{B} \Delta T\right)^{2}}\right],
$$

where $\lambda$ is the latent heat per atom for the addition of helium-rich fluid to a droplet, and $\Delta T$ is the supercooling. For a rough estimate of $\Delta T$, we equate the Jovian heat flux to $\rho b^{3} g H J$ :

$$
\begin{aligned}
\left(\frac{T}{\Delta T}\right)^{2} & \approx\left(\frac{2 \lambda^{2} k_{B} T}{\sigma^{3}}\right) \ln \eta, \\
\eta & =\left(\frac{H b^{3}}{a_{0}^{4}}\right) \epsilon_{0}^{-3 / 2},
\end{aligned}
$$

where $\epsilon_{0}{ }^{3 / 2}$ is the ratio of the heat flux to $\rho v_{s}{ }^{3}$. For Jupiter, $\epsilon_{0} \approx 10^{-8}$ and $\ln \eta \approx 100$. The theoretical calculations (Stevenson 1975) indicate that $\lambda \approx$ $0.5 k_{B} T$ at $T \approx 10^{4} \mathrm{~K}$, so we finally get $\Delta T / T \approx 10^{-2}$. If heterogeneous nucleation is possible, then the required superheating would be even smaller. If the supercooling becomes larger, then more droplets are nucleated and more energy is released, heating up the fluid. This acts as a servomechanism, keeping the supercooling at just the right level to supply the required energy output. In our subsequent analysis, we neglect $\Delta T$ relative to $T$. It is almost certainly small enough to ensure that nucleation rather than spinodal decomposition occurs (see Paper I).

Once helium separation has been initiated, three regions are formed (see Fig. $4 b$ ): (i) $P<P_{0}$ and $x(P)=x_{1}<x_{0}$; (ii) $P_{0}<P<P_{1}$ where $T_{\mathrm{ph}}[x(P), P]$ $\approx T(P)$; (iii) $P>P_{1}$ and $x(P)=x_{2}>x_{0}$. Regions (i) and (iii) are homogeneous and fully convective. The intermediate region is necessarily inhomogeneous because of the region of immiscibility. Consider, now, the life of a helium-rich droplet which nucleates out of the slightly supercooled mixture at $P=P_{0}, x=x_{1}$. According to Figure $4 b$, it has composition $x=x_{3}$. It eventually grows to about $1 \mathrm{~cm}$ size and begins to fall toward the center of the planet. Since diffusion times are much less than convective times, it will evolve along the right-hand-side boundary of the immiscibility region. At $P=P_{1}$, when the droplet has 
composition $x=x_{4}$, the droplet merges with the inner homogeneous region. However, it must continue to evolve along the phase boundary until it either reaches the critical point $\left(x=x_{c}\right)$ or the center of the planet. In Figure $4 b$ the most likely case is shown, in which the critical point is reached first. The droplet then evaporates, enriching the inner region with helium. During this phase of the evolution the inner region is being enriched with helium, but is still predominantly hydrogen.

Later in the evolution, the innermost hydrogenhelium region reaches the critical composition $x_{c}$. After this, helium-rich droplets fall all the way without evaporating, and a predominantly helium core must begin to form. This is indicated in Figure $4 c$. Notice that a well-defined density discontinuity exists at $P=P_{1}$. The negative slope of the phase diagram on the right side ensures that the predominantly helium core is homogeneously mixed.

Consider, now, the thermal structure of the inhomogeneous intermediate layer. The temperature drop $\Delta T$, and pressure drop $\Delta P$, across the layer are given by

$$
\begin{aligned}
& \Delta T=T_{\mathrm{ph}}\left(x_{2}, P_{1}\right)-T_{\mathrm{ph}}\left(x_{1}, P_{0}\right), \\
& \Delta P=P_{1}-P_{0} .
\end{aligned}
$$

Choice of $x_{1}$ (say) then gives a unique solution for the other parameters as a function of $d$, the layer thickness, given the phase diagram and the total helium content. The thermal and solute gradients can then be evaluated from equation (17). In the limit where $d \ll H_{p}$, we find

$$
\begin{aligned}
& \epsilon \approx 0.05\left(\frac{\Delta T}{T}\right)\left(\frac{H_{p}}{d}\right), \\
& \chi \approx 3 \Delta x\left(\frac{H_{p}}{d}\right),
\end{aligned}
$$

where $\Delta x=x_{2}-x_{1}$. For the metallic hydrogenhelium phase diagram (Stevenson 1975) we typically find $\Delta T / T \approx 10 \Delta x$ and $\epsilon \ll \chi$. This inequality arises because the fluid is degenerate and has a small thermal expansibility (i.e., $\left.\left|(\partial \ln \rho / \partial \ln T)_{x, p}\right| \ll 1\right)$. It would appear that unstable modes never exist for any layer thickness $d$. This could be misleading, however, since it does not take into account such nonlocal effects as "convective overshoot" (Gierasch 1971; Shaviv and Salpeter 1973) or the interaction of convection with the phase diagram itself.

Consider, for example, a fluid eddy of size $l$ moving upward with velocity $v_{c}$. This eddy impinges on the inhomogeneous layer from below, and begins to slow down as it loses buoyancy and penetrates the layer. The uppermost parts of this eddy are then helium-rich relative to the phase boundary composition, and helium droplets can nucleate and grow. We first evaluate the penetration of the eddy assuming that there is no nucleation. Its penetration distance $h$ can be found approximately by equating its initial kinetic energy to the work done against gravity in penetrating the lower density inhomogeneous layer:

$$
\rho v_{c}{ }^{2} l^{3} \approx \rho g_{\text {eff }} h^{2} l^{2},
$$

where $g_{\text {eff }}$ is the effective deceleration of the eddy:

$$
g_{\text {eff }}=-g h \frac{d x}{d z} .
$$

Thus,

$$
h \approx\left(\frac{v_{c}^{2} l}{g|d x / d z|}\right)^{1 / 3} .
$$

For $v_{c} \approx 10 \mathrm{~cm} \mathrm{~s}^{-1}, l \approx 10^{9} \mathrm{~cm}$ (the largest conceivable eddies in Jupiter, say), $g \approx 10^{3} \mathrm{~cm} \mathrm{~s}^{-2}$ and $|d x / d z| \approx 10^{-9} \mathrm{~cm}^{-1}$; we get $h \approx 10^{5} \mathrm{~cm}$. This means that "waves" of this amplitude exist at the transition between homogeneous and inhomogeneous layers. Regardless of nucleation, it follows that if the layer thickness is less than about $10^{5} \mathrm{~cm}$, then convective overshoot can transport heat through the layer.

Suppose, now, that the ambient fluid is on the verge of nucleation. Since nucleation is such a strong function of supercooling, nucleation would then begin immediately as the eddy began to penetrate the inhomogeneous layer. Droplets would grow at a rate limited by $D$, the helium diffusion coefficient (since heat diffusion is much more efficient). For $D \approx$ $10^{-3} \mathrm{~cm}^{2} \mathrm{~s}^{-1}$, droplets reach a size of $1 \mathrm{~cm}$ radius in $10^{3} \mathrm{~s}$. Since it takes $\sim 10^{4} \mathrm{~s}$ for the eddy to penetrate $h \approx 10^{5} \mathrm{~cm}$, these droplets begin to separate out before the eddy comes to rest. The droplet separation is inefficient, since the droplet velocity is only comparable to the convective velocity. Nevertheless, the theoretical calculations (Stevenson 1975) indicate that phase separation is accompanied by heating of the fluid (i.e., the latent heat is "positive"), so part of the eddy might become buoyant if it loses some of its helium. We shall now show that this instability does not in general occur, since it requires an unreasonably efficient separation process.

The uppermost portions of the eddy are helium-rich relative to the surrounding fluid by at most $h|d x / d z|=$ $\Delta x$. Suppose a fraction $\delta$ of this excess is completely eliminated by nucleation, growth, and removal of droplets. Since the latent heat is of order $k_{B} T$ per particle, the fluid is hotter than the surroundings by roughly $T \delta \Delta x$. Consequently, it is more dense than the surrounding fluid by $\Delta \rho$, where

$$
\frac{\Delta \rho}{\rho} \approx \Delta x \delta\left[\left(\frac{\partial \ln \rho}{\partial \ln T}\right)_{R, p}+(1-\delta)\left(\frac{\partial \ln \rho}{\partial x}\right)_{T, p}\right],
$$

where the second term arises because the fluid is still more helium-rich than the ambient medium. Since $(\partial \ln \rho / \partial \ln T)_{x, p} \approx-0.05$, whereas $(\partial \ln \rho / \partial x)_{T, p} \approx 2$, it follows that $\Delta \rho>0$ provided $\delta<0.97$, which is most likely.

In $\S$ II, the high-speed ejection of small volumes of fluid from one phase into the other during the recoil of an eddy was discussed for pure hydrogen. A similar effect probably occurs here, if the eddy is much larger 
than the thickness of the inhomogeneous region (so that gravity waves at the now diffuse "interface" would be possible). However, the application of equation (9) indicates that the amount of ejected fluid would have no significant effect on the distribution of thermal energy or helium.

We conclude that the inhomogeneous layer is probably stable with respect to convective overshoot or entrainment. Since the phase diagram (Paper I) predicts $\Delta T / T \approx 10 \Delta x$, equation (35) predicts $\epsilon \approx$ $0.1 \chi$. In Paper I, we found $(\kappa+\nu) \approx 0.5 \mathrm{~cm}^{2} \mathrm{~s}^{-1}$ and $(D+\nu) \approx 0.005 \mathrm{~cm}^{2} \mathrm{~s}^{-1}$, so $(\kappa+\nu) \epsilon>(D+\nu) \chi$ (eq. [24]) and the condition for overstability is satisfied. The criterion for layers (eq. [28]) may be marginally satisfied, but even if it is, the temperature gradient in the inhomogeneous layer will not differ greatly from that predicted for overstability. Overstable modes are inherently very inefficient, so the temperature gradient will be larger within the inhomogeneous layer than elsewhere. A consequence of this is that helium separation is accompanied by an increasing temperature in the innermost regions of the planet, despite the decreasing temperature externally. This is illustrated schematically in Figure 5.

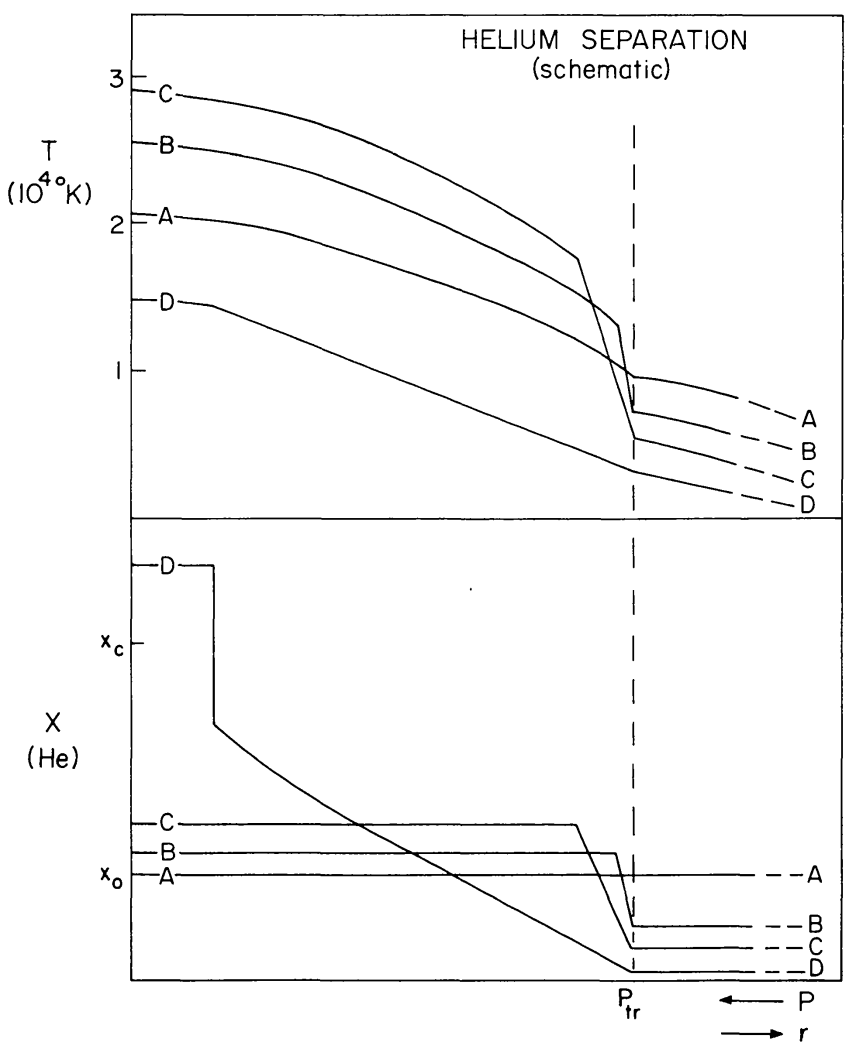

FIG. 5.-Temperature $T$ and helium composition $x$ as a function of pressure $P$ (or radial coordinate $r$ ) in a cooling hydrogen-helium planet. The curves $A, B, C, D$ are in order of increasing time. Note that in the early stages of helium separation, the central temperature increases as the external temperature decreases. Much later $(D)$, a helium core begins to form, and the temperature gradient in the inhomogeneous region decreases because the total internal heat flux is lower.
Assuming overstable modes, the thickness $d$ of the inhomogeneous layer can be estimated. For Jupiter, if we assume that the inner and outer helium fractions are $x_{2}=0.12$ and $x_{1}=0.06$, respectively, we find $d \approx$ $10^{8}-10^{9} \mathrm{~cm}$, a significant fraction of the planetary radius. (The precise value of $d$ depends on the efficiency of the overstable modes.) As the planet cools, the heat flux becomes less, and this layer becomes even thicker. The discussion of $\S$ IV indicates that convection above the inhomogeneous layer transports some helium upward, but this is always counteracted by nucleation.

To conclude, helium separation has the effect of prolonging the thermal evolution of the planet. Once it becomes thermodynamically favored, the separation proceeds with an efficiency that is neither very small nor very near $100 \%$. It leads to depletion of helium from the atmosphere, and a thermal structure that is substantially different from that of an adiabatic, homogeneous planet. An inhomogeneous layer is formed which is eventually stable with respect to largescale convective flows, and which can encompass a significant fraction of the planetary mass.

The effect of helium differentiation on the cooling rate of the planet can be large. We shall estimate this for the early stages of differentiation, where no predominantly helium-rich region has formed (case $B$, Fig. 5). The correct procedure for constructing an evolutionary sequence is to compare total (gravitational and internal) energies for a sequence of models with gradually decreasing effective temperatures. However, an examination of the calculations of Kiefer (1967) and Flasar (1973) indicates that the energy release from differentiation that is available for excess luminosity or heating of the planet can be adequately approximated as $Q_{\mathrm{Grav}}$, given by

$$
Q_{\mathrm{Grav}} \approx\left(\frac{d M}{d t}\right)_{\mathrm{He}} g H,
$$

where $(d M / d t)_{\mathrm{He}}$ is the rate at which a helium mass is moved down a distance $H$ in a gravity field $g$. In our case, $H$ is roughly the vertical separation of the centers of masses for the metallic and molecular fluids. Since differentiation increases the heat content of the core (even as the outer layers of the planet cool), we first consider what fraction of $Q_{\mathrm{Grav}}$ is required for this heating. Suppose the core composition changes from $x_{2}$ to $x_{2}+\Delta x_{2}$. The mass of helium required to do this is

$$
M_{\mathrm{He}} \approx \frac{4 \Delta x_{2} M_{c}}{\left(1-x_{2}\right)\left(1+3 x_{2}\right)},
$$

where $M_{c}$ is the mass of the core. We assume that the mass of the inhomogeneous layer is negligible (a good approximation during the early stages of evolution). The gravitational energy release is therefore

$$
E_{\mathrm{Grav}} \approx M_{\mathrm{He}} g H .
$$

However, $T_{2}$, the temperature at the boundary between the inhomogeneous layer and the metallic core, is related to $x_{2}$ according to the miscibility curve. Thus 
$T_{2}$ must change to $T_{2}+\Delta T_{2}$, where

$$
\Delta T_{2}=\left(\frac{d T}{d x}\right)_{\mathrm{ph}} \Delta x_{2} .
$$

According to the H-He phase diagram (Paper I),

$$
\left(\frac{d T}{d x}\right)_{\mathrm{ph}} \equiv T_{0} \approx 3 \times 10^{4} \mathrm{~K}
$$

for $x_{2} \approx 0.1$. The thermal energy increase of the (adiabatic) core, $E_{\mathrm{th}}$, is therefore

$$
E_{\mathrm{th}} \approx \gamma C_{v} T_{0} \Delta x_{2} M_{c},
$$

where $C_{v}$ is the specific heat per unit mass and $\gamma$ is the ratio of the average core temperature to the boundary temperature $T_{2}$. The ratio of $E_{\mathrm{th}}$ to $E_{\mathrm{Grav}}$ is therefore

$$
\frac{E_{\mathrm{th}}}{E_{\mathrm{Grav}}} \approx \frac{\gamma C_{v} T_{0}}{3 g H} \approx 0.2,
$$

assuming $x_{2} \approx 0.1, \gamma \approx 1.5, g \approx 3 \times 10^{3} \mathrm{~cm}^{2} \mathrm{~s}^{-1}$, $C_{v} \approx 2 \times 10^{8} \mathrm{ergs} \mathrm{g}^{-1}$, and $H \approx 4 \times 10^{9} \mathrm{~cm}$ for Jupiter. (Similar figures apply to Saturn.) We conclude that most of the energy release from differentiation must be radiated. The ratio above is an upper bound corresponding to highly inefficient heat transport through the inhomogeneous layer.

We proceed now to evaluate the cooling rate during differentiation. (Cooling rate is here defined to mean $d T_{e} / d t$, where $T_{e}$ is the effective temperature, since the total heat content of the planet may actually increase during the early stages of differentiation.) Let $T_{1}$ be the temperature at the boundary of the inhomogeneous region and the molecular envelope. We assume that $T_{1}$ and $T_{e}$ lie on the same adiabat, so that

$$
\frac{T_{1}}{T_{e}} \approx\left(\frac{P_{1}}{P_{e}}\right)^{n},
$$

where $P_{e}$ is the pressure at $T_{e}$ and $n$ is the average adiabatic index. As the helium differentiation proceeds, $P_{1}$ changes much less rapidly than $T_{1}$ and can be assumed to be constant. The transmission opacity of the atmosphere is also only slightly affected by a change in helium content (Trafton and Stone 1974) and the gravitational acceleration also changes little, so $P_{e}$ (eq. [5]) is approximately constant. The adiabatic index $n$ is affected significantly by the helium content (especially in the outermost layers) because helium is monatomic whereas hydrogen is diatomic. Since $n$ decreases as the helium content decreases, the decrease in $T_{e}$ during differentiation is actually less than it would be if $n$ were constant. (A change in $n$ also indirectly changes $P_{e}$ by changing the level in the atmosphere at which convective transport ceases to dominate.) Nevertheless, numerical calculations indicate that these effects are secondary and that $P_{1}, P_{e}$, and $n$ can all be considered constant in the first approximation. Equation (47) then implies

$$
\frac{d \ln T_{1}}{d t} \approx \frac{d \ln T_{e}}{d t}
$$

with a systematic error of typically $20-30 \%$.
Let $x_{1}$ be the composition of the molecular envelope. Conservation of helium implies $M_{c} \Delta x_{2} \approx-M_{\text {env }} \Delta x_{1}$, where $M_{\text {env }}$ is the mass of the molecular envelope. The gravitational energy release is therefore

$$
Q_{\mathrm{Grav}}-\frac{4 M_{\mathrm{env}}}{\left(1-x_{2}\right)\left(1+3 x_{2}\right) T_{0}} \frac{d T_{1}}{d t} g H .
$$

Equating $Q_{\mathrm{Grav}}=5 \times 10^{24} \mathrm{ergs} \mathrm{s}^{-1}$ for Jupiter and $T_{1}=10^{4} \mathrm{~K}$ implies (from eq. [47]) that $d T_{e} / d t \approx$ $-1.5 \mathrm{~K} / 10^{9} \mathrm{yr}$. In contrast, Hubbard's homogeneous, adiabatic model for Jupiter requires $d T_{e} / d t \approx-7 \mathrm{~K} /$ $10^{9} \mathrm{yr}$ for the present era (Hubbard 1977). For Saturn, equation (49) with $Q_{\mathrm{Grav}}=2 \times 10^{24} \mathrm{ergs} \mathrm{s}^{-1}$ implies $d T_{e} / d t \approx-1.3 \mathrm{~K} / 10^{9} \mathrm{yr}$, whereas homogeneous evolution requires 4 or 5 times more rapid cooling. Differentiation, once initiated, therefore has the effect of dramatically changing the luminosity-time relationship and increases the Kelvin time by a factor of 4 or 5 . In conjunction with the results of the homogeneous evolutionary calculations ( $\S \mathrm{II}$ ), these results suggest that Jupiter is not differentiating or at least has only recently (within the last $10^{9}$ years) begun differentiation, whereas Saturn may already have been differentiating for $\sim 2 \times 10^{9}$ years. If Saturn's luminosity is indeed $2 \times 10^{24}$ ergs s$^{-1}$ at present, then the simple model outlined above suggests that the molecular envelope (and atmosphere) have already been depleted by $20-30 \%$ of its primordial helium (i.e., from $x_{1} \approx$ 0.09 to $x_{1} \approx 0.07$ ).

The above calculations are applicable only if the molecular-metallic hydrogen transition is not firstorder. In the next section, we consider the additional complications that arise in determining the helium distribution when this restriction is relaxed.

\section{MORE GENERAL CASES}

In more general cases, both the first-order character of the molecular-metallic hydrogen transition and the limited solubility of helium in hydrogen must be considered. A qualitatively new feature is the Gibbs phase rule requirement that coexisting molecular and metallic phases must have different helium mass fractions. The discussion of Paper I indicates that helium would prefer to be mixed with molecular hydrogen. We consider in this section how that preference makes itself apparent in the helium distribution in a hydrogenhelium planet.

This section corresponds to Sector III of Figure 1. Both "hot" and "cold" starting points are considered because of the large uncertainty in $T_{c}\left(\mathrm{H}-\mathrm{H}_{2}\right)$. The designation "hot" or "cold" need not imply anything about the actual central temperature of the planet. For example, a "cold" starting point corresponds to an evolution in which the actual temperature was less than the critical temperature for the molecularmetallic hydrogen transition, when the pressure first exceeded a few megabars.

\section{a) The "Cold" Starting Point}

Consider a hydrogen-helium planet in its early evolution, when the pressure in the innermost hydrogen-helium region still has not reached several 


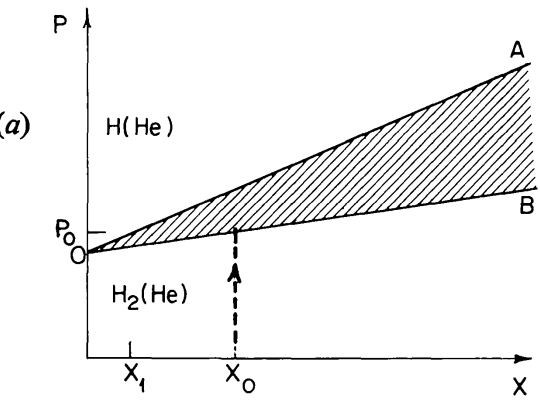

(c)
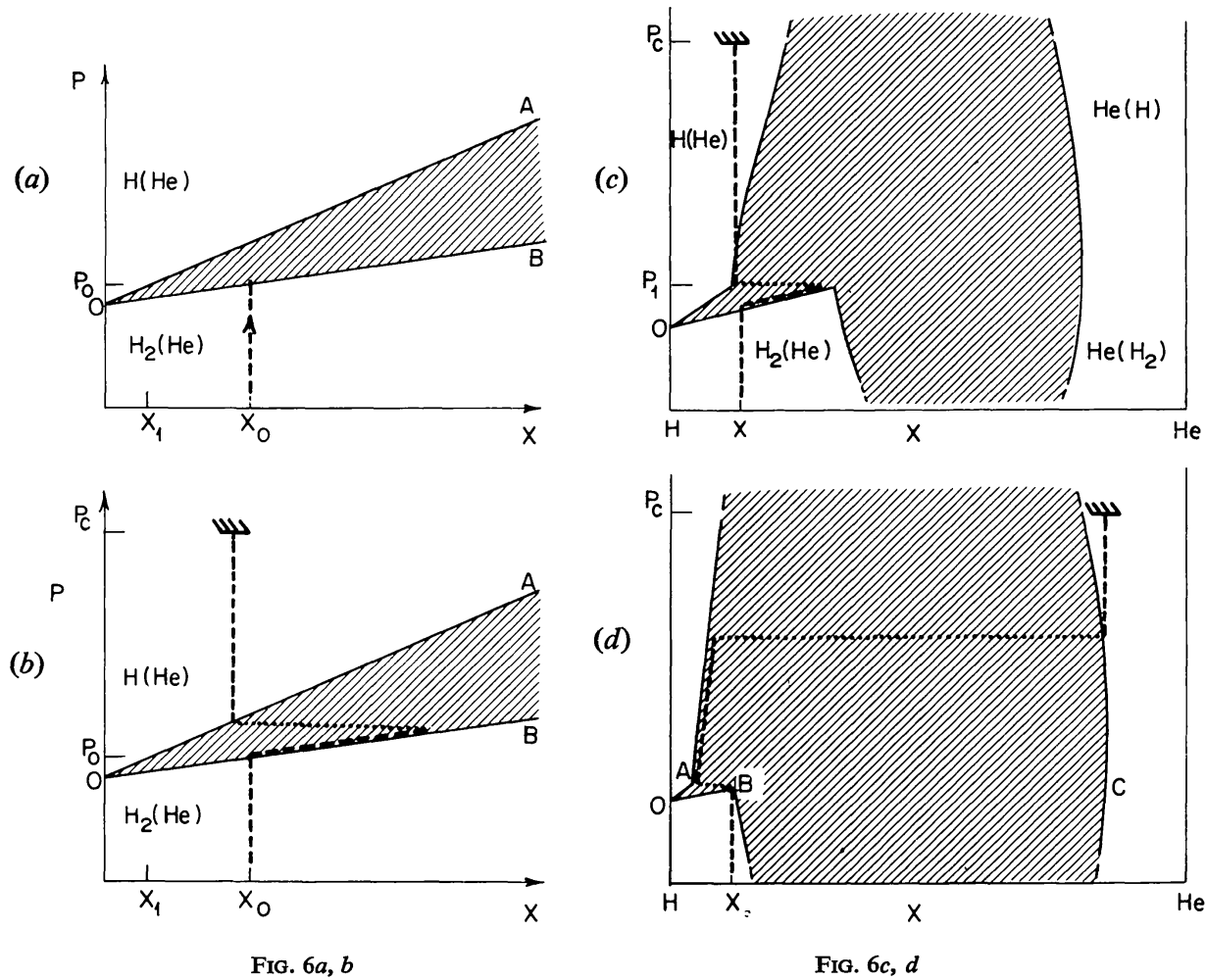

Fig. $6 c, d$

FIG. 6.-A sequence of phase diagrams for the "cold" stable case. These diagrams are slices of the three-dimensional $P, T, x$ diagrams, using the $P-T$ relationship actually existing within the planet. (The pressure coordinate $P$ can equally well be labeled by temperature.) The phase excluded region is shaded. The actual helium composition is represented by the dashed line. In $(a)$, the innermost hydrogen-helium fluid is just beginning to be compressed into the phase excluded region. Later, in $(b)$, an inhomogeneous molecular layer is formed on top of a homogeneous metallic layer. Subsequently, in $(c)$, the molecular fluid evolves into the triple point $B$, and helium-rich droplets form at $C$. An inhomogeneous metallic layer begins to form at $A$. Even later, at (d), the triple point composition becomes equal to $x_{0}$, and the entire molecular layer begins to be uniformly depleted of helium. The metallic hydrogen layer at $A$ is inhomogeneous, while a homogeneous helium-rich core forms in the innermost region.

megabars. We assume that the center of the planet is occupied by a small rocky core. This is a reasonable assumption from cosmogonic considerations (Podolak and Cameron 1974), but not crucial to our argument. As the planet continues to contract, the pressure increases and any given element of fluid evolves upward along the dashed line in Figure $6 a$. Eventually, in this "cold" case, a time is reached when the innermost hydrogen-helium fluid evolves into the phase excluded region (shaded in Fig. 6a). This occurs at $P=P_{0} \approx 3$ Mbar (see Paper I). Nucleation then becomes possible, and metallic droplets of lower helium content $\left(x=x_{1}\right)$ form and grow. Meanwhile, the molecular fluid becomes slightly helium-rich and evolves along the lower phase boundary. There are two very different cases, depending upon whether the helium-rich molecular phase is less or more dense than the helium-poor metallic phase.

Consider, first, the "stable" case in which the metallic phase is more dense. Once a macroscopic amount of this phase is formed, it settles into a layer covering the rocky core. The interface between this metallic layer and the molecular fluid is sharply defined, and lies exactly on the phase boundary for the relevant pressure. If no heat flux is transported through this interface, then the subsequent evolution is rather simple: the molecular fluid continues to evolve along the phase boundary toward a more helium-rich mixture. The metallic phase remains uniformly mixed, since the new fluid added to this phase is always a little more dense than the fluid already present. Figure $6 b$ shows the situation when the metallic-phase composition becomes almost the same as the original molecular-phase composition. A steady-state configuration is then reached in which subsequent contraction and compression effectively process molecular hydrogen into metallic hydrogen without changing the helium content. Only the rather thin intermediate molecular layer is inhomogeneous. Notice that the outer molecular layer retains its primordial helium content. We have, of course, assumed that the molecular phase still remains less dense than the metallic phase, even at $P=P_{1}$ in Figure $6 c$.

As the planet continues to cool, a time must be reached at which the molecular phase ceases to be less dense, or helium insolubility occurs. The former case is discussed later. In the latter case, the insolubility happens simultaneously in the molecular and metallic phases, as shown in Figure $6 c$. (This is a general thermodynamic principle and not a consequence of 
our phase diagram model.) Notice that the innermost molecular region evolves into a triple point. Droplets of helium-rich fluid nucleate from the molecular fluid at $B$ and the metallic fluid at $A$. These droplets form at $C$ and are more dense than either of the other coexisting phases. The growth and separation of these droplets then proceeds exactly as we discussed in $\S \mathrm{V}$. Notice that an inhomogeneous layer begins to form in the metallic layer, but the atmospheric helium content remains primordial still.

Even later in the evolution, the triple point evolves toward the primordial helium content, and the inhomogeneous molecular layer is eliminated by helium separation. Figure $6 d$ shows the point beyond which the atmosphere begins to be depleted in helium. The reason is that the innermost molecular region now begins to be depleted in helium relative to fluid above. This is an unstable situation, so the molecular layer remains fully mixed at the triple point composition, while the core becomes progressively more enriched. The triple point continues to evolve to lower helium fraction as the immiscibility region expands to fill more of $(P, T, x)$-space. The final (zero temperature) state is fully separated hydrogen above helium. If this case is applicable to Jupiter, then the current state of Jupiter is probably nearest to Figure $6 c$ : some helium separation may have occurred but there is no depletion from the atmosphere.

This rather simple picture can become more complicated when we consider (as we must) the transport of heat through the molecular-metallic boundary. We assume a constant, given heat flux which is determined by opacity considerations in the atmosphere, but which is ultimately derived from adiabatic contraction, or helium separation, or latent heat, or even radioactive heat from the rocky core. The question is whether the convective heat engine can do work transporting helium up into the atmosphere during the early evolution.

Return, now, to Figure $6 a$ where a metallic layer is just being formed, and the helium content of the molecular layer is beginning to be increased. In the presence of a fixed heat flux $F_{T}$, this is directly analogous to the situation we discussed in $\S I V$, in which solute is added at the lower boundary of a convecting fluid. Provided the solute is added sufficiently slowly, we found that it would all be convected upward. In our context, the criterion for complete mixing is that the work required to completely mix the helium upward be at most $(D / \kappa)^{1 / 2} F_{T}$, where $D$ and $\kappa$ are the helium and thermal diffusivities, respectively, for the molecular phase. If, as seems likely, electronic degrees of freedom are available for heat conduction (see Paper I), $D / \kappa \approx 10^{-2}$, so the upward mixing of helium will be rather inefficient. The actual amount of mixing depends on the value of $F_{T}$, which was surely many orders of magnitude larger during the early evolution than it is now (Graboske et al. 1975). The amount of work required to redistribute helium upward in Jupiter is not prohibitive even now. For example, the present internal heat flux of Jupiter acting for $10^{10}$ years could, in principle, supply energy suffi- cient to double the helium content in the molecular envelope of the planet (at the expense of the metallic core). However, the small value of $D / \kappa$ ensures that the actual amount of work done redistributing helium is small.

It seems likely, therefore, that the inhomogeneous layer (Fig. $6 b$ ) will form even in the presence of the heat flux. An additional complication can then arise: since the temperature gradient must be very large in the inhomogeneous layer (with the heat flux carried by inefficient, overstable modes), it is possible (and, in fact, quite likely) that the self-consistently determined phase boundary $O B$ in Figure $6 b$ no longer has a positive slope! This can occur if the latent heat for the pure molecular-metallic hydrogen transition is negative (in the sense discussed in $\S$ III). What then happens is that the dashed line in Figure $6 b$ ceases to follow the phase boundary but instead forms a purely diffusive-convective solution. Helium transport in or out of the metallic phase is then maintained by diffusion at the molecular-metallic interface. The inhomogeneous layer, the thickness of which was previously determined by the slope of the phase diagram, is then a few times $D / v_{b}$, where $v_{b}$ is the speed at which the molecular-metallic interface moves outward from the center of the planet. Typical values for Jupiter might be $D \approx 10^{-3} \mathrm{~cm}^{2} \mathrm{~s}^{-1}, v_{b} \approx 10^{-8} \mathrm{~cm}$ $\mathrm{s}^{-1}$, and $D / v_{b} \approx 10^{5} \mathrm{~cm}$. The upward transport of helium will then be close to the upper limit of $(D / \kappa)^{1 / 2} F_{T}$ in energy units.

We now discuss the case where the molecular phase, by virtue of its helium excess, ceases to be less dense than the coexisting metallic phase. The theoretical phase diagrams of Paper I indicate that this is quite likely. We suppose that the early evolution is as in the stable case, but that somewhere between the Figure $6 a$ and Figure $6 b$, the densities of the coexisting phases become equal. The planet continues to contract, so that at time $t$ later, there exists a thin molecular layer of thickness $v_{\mathrm{ev}} t$, which is more dense than the metallic fluid immediately beneath it. Here $v_{\mathrm{ev}}$ is a velocity characterizing the evolution rate, and is comparable to the velocity of the molecular-metallic boundary relative to the center of the planet.

A Rayleigh-Taylor instability is now possible. The time that disturbances of wavelength $\lambda$ take to attain an amplitude $\sim \lambda$ is (Chandrasekhar 1961)

$$
\tau_{R T} \approx \frac{4 v \rho}{g \lambda \Delta \rho},
$$

where $\nu$ is the kinematic viscosity and $\Delta \rho$ is the density difference between the overdense molecular layer and the metallic fluid. Clearly,

$$
\begin{aligned}
\frac{\Delta \rho}{\rho} & \approx \frac{v_{\mathrm{ev}} t}{H_{p}}, \\
\lambda & \approx v_{\mathrm{ev}} t,
\end{aligned}
$$

since only the layer of thickness $v_{\mathrm{ev}} t$ can participate in the instability. Equating $t$ to $\tau_{R T}$ gives the time 
for breakup of the layer:

$$
t \approx\left(\frac{\nu H_{p}}{g v_{\mathrm{ev}}^{2}}\right)^{1 / 3}
$$

For $v_{\mathrm{ev}} \approx 10^{-4} \mathrm{~cm} \mathrm{~s}^{-1}, t \approx 10^{5} \mathrm{~s}$ and $\lambda \approx 10 \mathrm{~cm}$. For $v_{\mathrm{ev}} \approx 10^{-9} \mathrm{~cm} \mathrm{~s}^{-1}$ (a present-day value for the motion of the interface in Jupiter), $t \approx 10^{9} \mathrm{~s}$ and $\lambda \approx 0.1 \mathrm{~cm}$. Thus the instability is typically characterized by the breakup of a very thin layer of fluid into droplets of size $1 \mathrm{~cm}$, to within an order of magnitude or 2. The helium diffusion time for such droplets is small (about $10^{3} \mathrm{~s}$ ) relative to the time they would take to fall a substantial fraction of a scale height, so these droplets remain in equilibrium with the phase boundary as they fall under gravity. They evolve in the direction of the arrow in Figure 7, becoming progressively more dense than the metallic phase. For the choice of phase diagram in Figure 7, these droplets merge in a helium-rich inner region at $P>P_{2}$. The helium-poor metallic region is shown as homogeneous in Figure 7, but it may actually tend to become stably stratified (with more helium in the innermost regions), for two reasons. First, the helium-rich and helium-poor fluids at $P=P_{2}$ are not in phase coexistence: there is a chemical potential difference tending to drive helium upward into the helium-poor fluid. Second, the shaded forbidden region in Figure 7 is actually expanding as the planet cools, so helium-poor droplets may nucleate from the helium-rich fluid and rise to merge with the helium-poor fluid above. These effects will not stop convection in the entire helium-poor layer $\left(P_{1}<P<\right.$ $P_{2}$ ), but rather lead to a diffusive-convective solution of the type discussed in $\S I V$. Except for a diffusive layer near $P=P_{2}$, most of the helium-poor layer continues to convect and transport some helium upward.

The subsequent evolution in this case is actually not much different from the stable case. The shaded

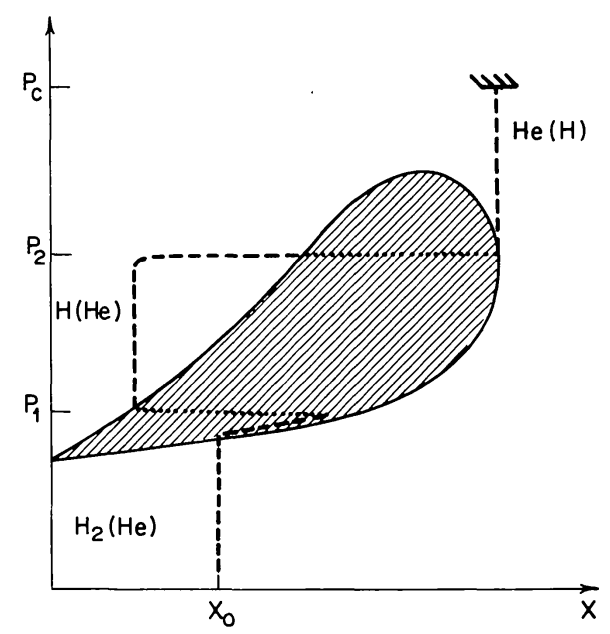

FIG. 7.-The unstable "cold" case. The coexisting phases at $P=P_{1}$ have the same density. Droplets break away from the molecular fluid at $P=P_{1}$, and evolve in the direction of the arrow to merge with the helium-rich core at $P=P_{2}$. Subsequent evolution of this figure is similar to Fig. 6 . region in Figure 7 will expand and form a diagram somewhat like Figure $6 c$. The molecular fluid at $P=$ $P_{1}$ will then eventually evolve into the triple point. The situation will then be similar to Figure $6 c$, except that (a) a predominantly helium core has already formed, (b) the helium-poor metallic layer above this helium core will have a lower helium content than the primordial mixture, and $(c)$ the coexisting phases at $P=P_{1}$ have the same density.

The equality of densities at the molecular-metallic interface leads to another novel feature: large-amplitude gravity waves excited by convection. In Salpeter and Stevenson (1976), interfacial gravity waves were found to have small amplitude at a pure molecularmetallic interface, because of the substantial density difference between the phases. In the case where the densities are equal, however, the amplitude of the waves is limited only by the lower compressibility of the metallic phase relative to the molecular phase. Let $\Delta z$ be the distance measured upward from the equaldensity interface. The densities of the two phases (one stable, the other metastable) at this position are

$$
\begin{aligned}
& \rho_{\mathrm{met}} \approx \rho_{0}\left(1-\alpha \Delta z / H_{p}\right), \\
& \rho_{\mathrm{mol}} \approx \rho_{0}\left(1-\beta \Delta z / H_{p}\right),
\end{aligned}
$$

for the metallic and molecular fluids, respectively. The values of $\alpha$ and $\beta$ are determined mainly by the properties of the pure hydrogen phases, rather than by the helium admixture, and are roughly $\alpha \approx 0.45$, $\beta \approx 0.55$. Consider an eddy of metallic fluid with velocity $v_{c}$ and size $l$ incident on the interface. It penetrates a distance $h$ given by

$$
\rho v_{c}^{2} l^{3} \approx \rho g(\beta-\alpha)\left(h / H_{p}\right) h^{2} l^{2} .
$$

For simple mixing-length theory, $v_{c} \approx 10^{-6} v_{s}\left(l / H_{p}\right)^{1 / 3}$, whence we find

$$
\frac{h}{l} \approx 10^{-4}\left(\frac{H_{p}}{l}\right)^{4 / 9},
$$

so $h \geqslant l$ (i.e., wavelength exceeds wave amplitude) for $l \leqslant 10^{2} \mathrm{~cm}$. At this size, molecular viscosity is not yet important, so it is possible for drops of size $\sim 10^{2} \mathrm{~cm}$ to break away from the interface and proceed a few times their own length into the opposite phase. Longer wavelengths have larger amplitude but are comparatively stable $(h / l<1)$.

Despite the larger distortions of the interface in this case, the interface will still not be completely destroyed. In other words, the considerations of Salpeter and Stevenson (1976; also see § III) still apply, and the interface is "isothermal."

\section{b) The "Hot" Starting Point}

We now consider a case in which the influence of phase transitions occurs much later in the evolution of the planet. Figure $8 a$ shows one particularly likely situation in which the phase-excluded region begins small and then expands until it comes in contact with the actual (homogeneous) helium distribution at some 

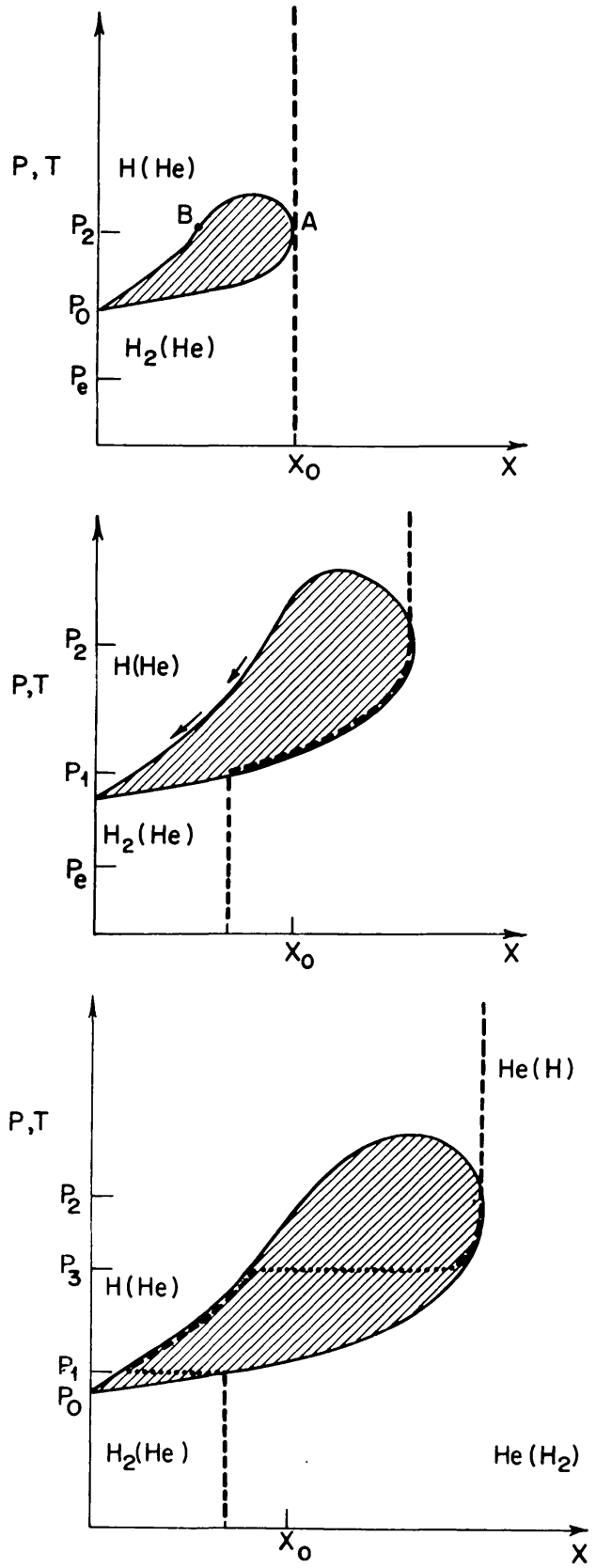

FIG. 8.-The "hot" case. The dashed line represents the helium concentration, and the phase excluded region is shaded. In $(a)(t o p)$, helium-poor droplets $(B)$ nucleate from the fluid at $A$ and rise along the phase diagram as indicated by the arrow. These droplets eventually become pure metallic hydrogen, then evaporate at $P_{e}<P<P_{0}$. In $(b)$ (middle), the subsequent evolution dilutes the helium content of the molecular envelope, while a helium-rich region forms. In $(c)$ (bottom), helium-poor metallic droplets at $P=P_{1}$ no longer have lower density than helium-rich molecular fluid, so a metallic layer forms. The final state is not shown since it is equivalent to Fig. $6 d$.

pressure $P=P_{2}$. From the fluid at $A$, helium-poor metallic fluid droplets nucleate at $B$. These droplets are always less dense for any plausible phase diagram like Figure $8 a$, so they begin to rise, maintaining equilibrium with the phase boundary, as shown by the arrow in Figure $8 a$. As usual (cf. $\S \mathrm{V}$ ), the droplets never grow much larger than $1 \mathrm{~cm}$ radius before fragmenting. The droplets evolve to become essentially pure metallic hydrogen at $P=P_{0}$. They can now change phase, mainly by evaporation at the droplet surface, but also by nucleation within the droplet. In either case, the rate at which the droplet converts back to the molecular phase is determined by latent heat considerations. We shall not discuss the details of this, but we assume that the resulting dilution of the molecular fluid at $P \approx P_{0}$ is sufficiently delocalized that convection maintains compositional uniformity. Presumably, microscopic droplets of metallic hydrogen have a very long lifetime, but even they cannot rise to pressures lower than $P_{e}$, the pressure at which the density of the droplet is the same as the ambient medium, unless they are transported by convection. A steady-state metastable metallic hydrogen "mist" presumably exists, perhaps to quite low pressures, because of convective transport.

As the phase excluded region expands toward larger $x$, the region $P>P_{2}$ remains fully mixed since the region near $P=P_{2}$ is being continually enriched in helium. Above this layer, an inhomogeneous molecular layer forms. At even lower pressures, a homogeneous layer, extending up to the atmosphere, exists. This layer has a diluted composition relative to primordial, because of the continuous addition of pure metallic hydrogen droplets. This is illustrated in Figure $8 b$.

The homogeneous molecular layer cannot evolve all the way to pure hydrogen because some level (labeled $P=P_{1}$ in Fig. $8 b$ ) exists at which the coexisting phases now have equal density. Helium-poor metallic droplets at $P=P_{1}$ no longer rise, and begin to form a layer separating two molecular regions. This is shown in Figure $8 c$. There are now two interfaces, at $P=P_{1}$ and at $P=P_{3}$. The interface at $P=P_{1}$ is approximately a constant-derísity interface. It is rather unstable, since pieces of the metallic phase could break away and become buoyant by losing their helium as they continue to evolve along the phase boundary. The actual dynamic steady state presumably has the interface slightly displaced from the equal density level, so as to ensure greater stability. The discussion earlier in this section on waves at a constant-density interface indicates that the instability is not catastrophic.

The subsequent evolution is then quite straightforward. Eventually an inflection develops in the molecular phase boundary in Figure $8 c$, and the phase excluded region evolves toward a diagram such as Figure $6 d$. The helium distribution would then be the same as in the "cold" evolution. Thus the final state is similar for "hot" and "cold" starting points, but the paths by which this state is reached are different.

\section{DISCUSSION AND CONCLUSION}

In Figure 1, the entire evolution of a hydrogenhelium planet can be characterized as a semi-infinite line segment, the extension of which passes through the origin. We first summarize in qualitative fashion the 
six possible evolutions corresponding to possible hightemperature starting points in Figure 1. Some of these evolutionary sequences also have further alternatives, depending on the relative densities of coexisting molecular and metallic phases.

Sector I (hot).-As the planet cools down, helium begins to separate out. At first, a somewhat enriched metallic region and a somewhat depleted molecular region exist, separated by an inhomogeneous layer. Later, a predominantly helium core begins to form.

Sector I (cold). - During the early evolution, helium begins to separate out and (probably) forms a predominantly helium core. Depletion of helium from the atmosphere then begins very early in the evolution of the planet.

Sector III (hot).-As the planet cools down, heliumpoor metallic droplets nucleate from the mixture, rise, and eventually lead to the dilution of the atmospheric helium abundance. At first, a helium-poor molecular layer and a helium-enriched inner region exist, separated by an inhomogeneous layer. Later, an inhomogeneous metallic layer also begins to form, while the inner region slowly evolves toward a predominantly helium composition.

Sector III (cold).-(a) Stable: If the metallic phase is more dense than the coexisting molecular phase, then initially an inhomogeneous molecular layer is formed, separating homogeneous molecular and metallic layers of essentially primordial composition. A small amount of helium is transported upward into the uniformly mixed molecular envelope. Later, helium separation begins in the metallic layer and the inhomogeneous molecular layer. Much later still, helium begins to be depleted from the homogeneous molecular envelope, and a predominantly helium core begins to form. (b) Unstable: If the metallic phase becomes less dense than the coexisting molecular phase, then formation of a helium core (or helium-enriched inner region) proceeds immediately, usually by depleting helium from the metallic phase. Subsequent evolution is similar to the stable case, except that the molecular-metallic interface has no density discontinuity.

Sector II (hot).-This intermediate regime is difficult to characterize since it combines the effects of Sectors I and III. A typical sequence of events would be that helium-poor metallic droplets nucleate from the mixture and rise to dilute the molecular envelope. Soon after, the helium-enriched inner region begins to phaseseparate. Subsequently, there can be as many as three inhomogeneous regions and four interfaces. These complexities arise because Sector II corresponds to a coincidental similarity of the values of $T_{c}\left(\mathrm{H}-\mathrm{H}_{2}\right)$ and $T_{c}(\mathrm{H}-\mathrm{He})$.

Sector II (cold).--Similar complexities to the "hot" case. Figure 9 shows one possible helium distribution.

The complications of Sector II are not of concern except when $T_{c}\left(\mathrm{H}-\mathrm{H}_{2}\right)$ is fortuitously very similar to $T_{c}(\mathrm{H}-\mathrm{He})$.

It is evident that detailed numerical calculations are premature at this stage. To give an indication of the impact of our considerations on the thermal history of a hydrogen-helium planet, we shall consider how

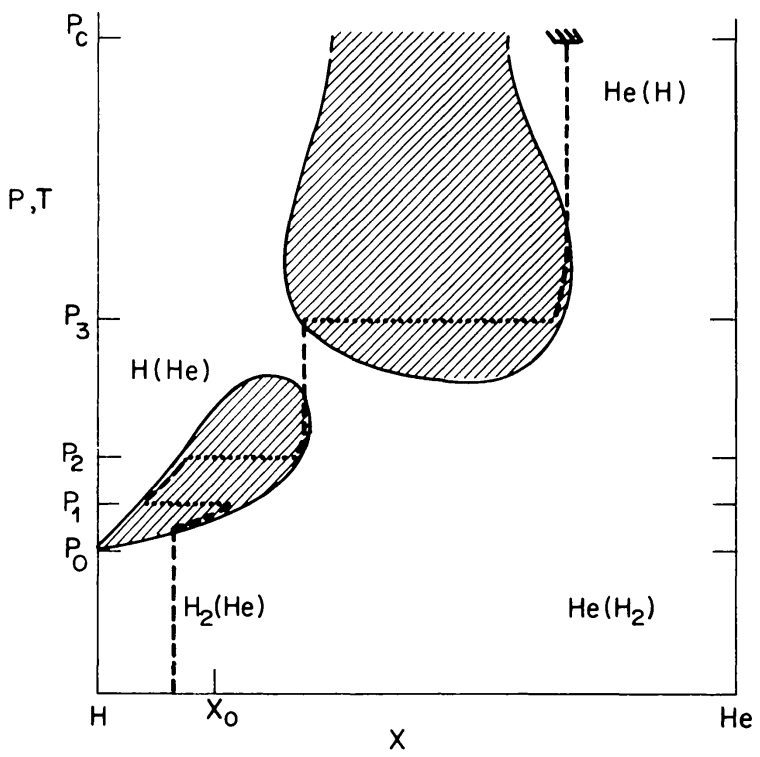

FIG. 9.-An intermediate case (Sector II of Fig. 1, cold starting point). This is essentially the sum of Fig. $4 c$ and Fig. $8 c$.

the homogeneous, evolutionary models of Jupiter (Graboske et al. 1975; Hubbard 1977) are modified, using several possible choices of $T_{c}\left(\mathrm{H}-\mathrm{H}_{2}\right)$, but for $T_{c}(\mathrm{H}-\mathrm{He})=12,000 \mathrm{~K}$ and $T_{c}\left(\mathrm{H}_{2}-\mathrm{He}\right)=6000 \mathrm{~K}$. Our considerations may actually be more relevant to Saturn, but we choose Jupiter because it is better understood and better constrained by current observations. We shall also neglect latent heat effects, since helium redistribution generally has the dominant effect on the planetary cooling rate.

In the homogeneous cooling models of Jupiter, models for the very early evolution are very speculative, and hydrodynamic effects may be important (Bodenheimer 1974), but this is of no concern here, since we consider only the evolution subsequent to the planetary center becoming degenerate $(P \geqslant 1 \mathrm{Mbar}$ central pressure). The central temperature is then at most about $50,000 \mathrm{~K}$, and the planet is probably only about $10^{6}$ years old.

Consider, first, $T_{c}\left(\mathrm{H}-\mathrm{H}_{2}\right)=60,000 \mathrm{~K}$. In this case we have a "cold" starting point, and the first-order character of the molecular metallic transition is encountered as the center first becomes degenerate. The phase diagram (Paper I) suggests that the unstable case is probably appropriate, so a helium-rich core immediately begins to form and grow at the expense of a helium-depleted metallic hydrogen region (Fig. 7). The gravitational energy release would prolong the high-luminosity phase of Jupiter, but since this phase lasts only a short time, it would not greatly affect the "age" (i.e., the time taken to reach the observed excess luminosity). Nevertheless, the age is substantially affected since the phase excluded region in Figure 7 continues to expand throughout the evolution, and the helium core becomes progressively more heliumrich. The molecular envelope retains its primordial 
helium abundance and is even slightly enriched with helium by upward convective transport. If the effective temperature is decreased, then temperatures in the deep interior are decreased by a comparable fractional amount and the excluded region in Figure 7 expands slightly. A rough calculation, analogous to that in $\S \mathrm{V}$, indicates that the rate of cooling is substantially less than that for a homogeneous planet, because of the continuing helium differentiation. (Unlike the simple calculation in $\S \mathrm{V}$, a precise calculation is difficult, since it necessarily depends on the efficiency of heat transport through the inhomogeneous layer.) In other words, if upward convective transport of helium and latent heat effects are both negligible, then the present state of Jupiter is not compatible with $T_{c}\left(\mathrm{H}-\mathrm{H}_{2}\right)=$ $60,000 \mathrm{~K}$ unless Jupiter is much older than $4.5 \times 10^{9}$ years.

Consider, now, $T_{c}\left(\mathrm{H}-\mathrm{H}_{2}\right)=20,000 \mathrm{~K}$. The initial central temperature of $50,000 \mathrm{~K}$ then corresponds to a "hot" starting point in Sector III of Figure 1. Over $10^{9}$ years elapse before the situation in Figure $8 a$ occurs. Helium-poor metallic droplets then form, and rise to lower pressures to dilute the molecular layer above. In this case, the present state of Jupiter would have a helium-rich core which joins continuously with an inhomogeneous molecular layer and ultimately with a helium-poor molecular envelope. The atmosphere would be depleted of helium, but no density discontinuity would exist anywhere in the planet (until much later in the evolution-about $10^{10}$ years from now). This is essentially as illustrated in Figure $8 b$. The gravitational energy released, integrated luminosity, and central temperature would all be larger than in an adiabatic, homogeneous model. Once again, it is clear that if helium differentiation is in progress, then the cooling rate would be much slower than for a homogeneous model, and the present luminosity o Jupiter would only be consistent with an age in excess of $5 \times 10^{9}$ years. Nevertheless, $T_{c}\left(\mathrm{H}-\mathrm{H}_{2}\right) \leqslant 20,000 \mathrm{~K}$ is consistent with observations, when allowance is made for all the uncertainties.

Consider, finally, $T_{c}\left(\mathrm{H}-\mathrm{H}_{2}\right)=0 \mathrm{~K}$. In this case, the adiabatic, homogeneous evolutionary models are correct until immiscibility begins in the helium fluid (see $\S \mathrm{V}$ ). In Jupiter, immiscibility may have begun within the last $10^{9}$ years, or is about to begin within about $10^{9}$ years.

Similar comments apply to Saturn, but with a lesser degree of certainty. Present-day temperatures in Saturn's interior are lower than those at comparable pressures in Jupiter by perhaps $20 \%$ (see, for example, Podolak and Cameron 1974). Immiscibility has probably already been encountered, and this is an attractive explanation for the observed anomalously large excess luminosity (Pollack et al. 1977). A possible (but less likely) alternative is that the molecular-metallic transition is first-order in Saturn, but not in Jupiter. (These conclusions assume that current estimates of the Saturnian excess luminosity are reliable.)

In the preceding discussion we have not tried to keep account of the various latent heat effects associated with the various transitions and layer formations. We predict (on the basis of the discussion of $\S$ III, and extensions thereof) that the following rules will apply: (i) In homogeneous layers, the temperature gradient is essentially adiabatic. (ii) In inhomogeneous layers, the temperature gradient appropriate to overstable modes probably applies. (iii) At each interface, the temperature (and not the entropy) is continuous. (iv) No "twophase" regions exist near first-order phase transitions (i.e., transitions are "abrupt").

These rules provide a unique prescription for evaluating the temperature everywhere.

We proceed, now, to a brief consideration of the distribution of minor constituents (such as water). In Paper I ( $\S$ VI) the partitioning of minor constituents among the various hydrogen-helium phases was discussed, but purely from a thermodynamic view. Thermodynamic equilibrium may not be achieved for two reasons. First, in the growth of droplets from a nucleation seed, any species which diffuses much more slowly than helium would not achieve equilibrium partitioning if the droplet moves to a region of substantially different thermodynamic environment during one diffusion time. For typical parameter values $(\S \mathrm{V})$, droplets are $1 \mathrm{~cm}$ in radius and move at $\sim 10 \mathrm{~cm} \mathrm{~s}^{-1}$. Except in special cases (such as at the beginning of differentiation), a droplet would have to move $10^{8} \mathrm{~cm}$ or more to encounter a substantially different environment. Nonequilibrium partitioning would therefore require a diffusivity less than $\sim 10^{-6} \mathrm{~cm}^{2} \mathrm{~s}^{-1}$. This is unlikely in the fluid state (the helium diffusivity is $\sim 10^{-3} \mathrm{~cm}^{2} \mathrm{~s}^{-1}$, and larger molecules would not diffuse more than about one order of magnitude more slowly). The second and more important cause of nonequilibrium is the difficulty that we have already considered for helium: upward convective transport in cases where the solute would prefer to be mixed with the molecular phase (a likely situation, according to Paper I). If, as is likely, the solute diffuses less rapidly than helium, then it tends to be trapped in the helium diffusive layer (see $\S$ IV) which forms at interfaces. Any solute that diffuses more rapidly than helium probably achieves a distribution close to thermodynamic equilibrium. Unlike helium, the redistribution of minor constituents is not fundamentally limited by energy considerations (the convective heat engine could in principle transport several tens of Earth masses of material from the center to the atmosphere of Jupiter in less than $4.5 \times 10^{9}$ years). However, dynamic considerations may preclude efficient redistribution, just as they did for helium.

Nevertheless, any process which redistributes helium will also redistribute minor constituents. The considerations of Paper I ( $(\mathrm{VI})$ indicate that $\mathrm{H}_{2} \mathrm{O}, \mathrm{NH}_{3}$, and $\mathrm{CH}_{4}$ probably prefer molecular and helium-rich phases. An observational test of the considerations of this paper would be accurate determinations of the atmospheric compositions of the giant planets, especially Saturn. Unfortunately, the interpretation of such data is likely to be ambiguous.

We conclude by noting some of the inadequacies in the present analysis. First and foremost, our analysis lacks quantitative predictive power because the critical 
temperature of the molecular-metallic hydrogen firstorder transition is not known to better than an order of magnitude. Further quantitative progress in the latent heats of transition and the molecular hydrogenhelium miscibility gap is also needed. Until these parameters are known, no detailed evolutionary model of Jupiter or Saturn can be very reliable. (Conversely, evolutionary calculations may be useful in imposing constraints on the various poorly known parameters.)

Numerous assertions made in this paper about the properties of convection in turbulent, inhomogeneous fluids must be regarded as nonrigorous. Even if we knew the hydrogen-helium phase diagram exactly, our predictions could be subject to error, simply because we may have overlooked some convective mode or instability.

Notwithstanding these admissions of ignorance, the following conclusions are indicated:

1. The major cause of deviations from homogeneous, adiabatic evolution is helium differentiation. Latent heat effects (either contemporary or primordial) are likely to be much less important. (It is not possible to have latent heat effects without some helium differentiation and vice versa.)

2. Helium differentiation can occur either because of immiscibility or because of the required discontinuity in helium fraction at a first-order molecularmetallic hydrogen transition.
3. Regardless of the cause of differentiation, it is almost invariably an ongoing process which, once initiated, has a dominant effect on the cooling rate of the planet for all subsequent time.

4. The assumed age and known luminosity of Jupiter indicate that helium differentiation began $\leqslant 10^{9}$ years ago, or will begin in $\leqslant 10^{9}$ years from the present time. This implies that the critical temperature $T_{c}\left(\mathrm{H}-\mathrm{H}_{2}\right)$ cannot greatly exceed $20,000 \mathrm{~K}$.

5 . The assumed age and known luminosity of Saturn indicate that differentiation may have been proceeding for $2 \times 10^{9}$ years already, but the uncertainties are large and this conclusion is necessarily tentative.

6. Helium differentiation is accompanied by a comparable (or even greater) redistribution of minor constituents. This may provide an observational test of our theory.

We wish to thank P. Gierasch, W. B. Hubbard, R. Smoluchowski, and J. S. Turner for discussions. Support by National Science Foundation grants AST 75-21153 and MPS 74-17838 and National Aeronautics and Space Administration grant NGR 33 $010-188$ is gratefully acknowledged.

\section{REFERENCES}

Anderson, J. D., Hubbard, W. B., and Slattery, W. L. 1974, Ap. J. (Letters), 193, L149.

Aumann, H. H., Gillespie, C. M., Jr., and Low, F. J. 1969, Ap. J. (Letters), 157, L69.

Bodenheimer, P. 1974, Icarus, 23, 319.

Busse, F. H. 1976, Icarus, 29, 255.

Busse, F. H., and Schubert, G. 1971, J. Fluid Mech., 46, 801.

Caldwell, D. R. 1974, J. Fluid Mech., 64, 347.

Cameron, A. G. W. 1973, Space Sci. Rev., 15, 121.

Chandrasekhar, S. 1961, Hydrodynamics and Hydromagnetic Stability (New York: Oxford University Press), chap. 4.

Clayton, D. D. 1968, Principles of Stellar Evolution and Nucleosynthesis (New York: McGraw-Hill), p. 138.

Feder, J., Russell, K., Lothe, J., and Pound, G. 1966, Adv. in Phys., 15, 111.

Flasar, F. M. 1973, Ap. J., 186, 1097

Gierasch, P. 1971, J. Atm. Sci., 22, 315.

Gierasch, P., and Stevenson, D. J. 1977, unpublished.

Graboske, H. C., Pollack, J. B., Grossman, A. S., and Olness, R. J. 1975, Ap. J., 199, 265.

Gulkis, S., and Poynter, R. 1972, Phys. Earth Planet. Inter., 6, 36 .

Hide, R. 1974, Proc. Roy. Soc. London, A366, 63.

Howard, L. N. 1964, in Proc. 11th Int. Congress Applied Mechanics (Berlin: Springer-Verlag), p. 1109.

Hubbard, W. B. 1968, Ap. J., 152, 745. 1973, Ap. J. (Letters), 182, L35. 1977, Icarus, 30, 305.

Hubbard, W. B., and Slattery, W. L. 1976, in Jupiter, ed. T. Gehrels (Tucson: University of Arizona Press), p. 176.

Hubbard, W. B., and Smoluchowski, R. 1973, Space Sci. Rev., $14,599$.

Ingersoll, A. P., Münch, G., Neugebauer, G., and Orton, G. S. 1976, in Jupiter, ed. T. Gehrels (Tucson: University of Arizona Press), p. 197.

Jeffreys, H., and Jeffreys, B. S. 1950, Methods of Mathematical Physics (2d ed.; Cambridge: Cambridge University Press), chap. 20.

Kiefer, H. H. 1967, J. Geophys. Res., 72, 3179.
Landau, L., and Lifshitz, E. M. 1959, Fluid Mechanics (Reading, MA: Addison-Wesley), p. 171.

Lang, N., and Kohn, W. 1970, Phys. Rev., 113, 4555.

Linden, P. F. 1973, J. Fluid Mech., 60, 467. . 1974, Deep Sea Res., 21, 283.

Linden, P. F., and Shirtcliffe, T. G. L. 1976, unpublished.

Long, R. R. 1975, J. Fluid Mech., 70, 305.

Nolt, I. G., Radostitz, J. V., Donnelly, R. J., Murphy, R. E., and Ford, H. C. 1974, Nature, 248, 659.

Podolak, M. 1977, Icarus, 30, 155.

Podolak, M., and Cameron, A. G. W. 1974, Icarus, 22, 123. . 1975, Icarus, 25, 627.

Pollack, J. B., Grossman, A. S., Moore, R., and Graboske, H. C. 1977, Icarus, 30, 111

Rieke, G. 1975, Icarus, 26, 37.

Salpeter, E. E. 1973, Ap. J. (Letters), 181, L83.

Salpeter, E. E., and Stevenson, D. J. 1976, Phys. Fluids, 19, 502.

Schubert, G., Turcotte, D. L., and Oxburgh, E. R. 1970, Science, 169, 1075.

Shaviv, G., and Salpeter, E. E. 1973, Ap. J., 184, 191.

Shirtcliffe, T. G. L. 1967, Nature, 213, 489.

Smoluchowski, R. 1967, Nature, 215, 691.

Spiegel, E. A. 1972, Ann. Rev. Astr. Ap., 10, 261.

Stevenson, D. J. 1974, Icarus, 22, 403. 1975, Phys. Rev., 12B, 3999

1976, Ph.D. thesis, Cornell University.

Stevenson, D. J. 1977, in The Origin of the Solar System, ed. S. F. Dermott (New York: Wiley).

Stevenson, D. J., and Ashcroft, N. W. 1974, Phys. Rev., 9A, 782.

Stevenson, D. J., and Salpeter, E. E. 1976, in Jupiter, ed. T. Gehrels (Tucson: University of Arizona Press), p. 85. 1977, Ap. J. Suppl., 35, 221 (Paper I).

Trafton, L. M., and Stone, P. H. 1974, Ap. J., 188, 649.

Turcotte, D. L., and Schubert, G. 1971, J. Geophys. Res., 76, 7980.

Turner, J. S. 1967, Deep Sea Res., 16, 497.

ㄴ. 1968a, J. Fluid Mech., 33, 183. 
Turner, J. S. 1968b, J. Fluid Mech., 33, 639.

Turner, J. S., and Stommel, H. 1964, Proc. Nat. Acad. Sci. $52,49$.

E. E. SALPETER: Newman Laboratory of Nuclear Studies, Cornell University, Ithaca, NY 14853

D. J. Stevenson: Research School of Earth Sciences, ANU, P.O. Box 4, Canberra 2600, Australia
Walin, G. 1964, Tellus, 16, 389.

Zharkov, V. N., and Trubitsyn, V. P. 1976, in Jupiter, ed. T. Gehrels (Tucson: University of Arizona Press), p. 133. 